\title{
Parabolic type equations associated with the Dirichlet form on the Sierpinski gasket
}

\author{
Xuan Liu ${ }^{1} \cdot$ Zhongmin Qian $^{2}$ (]) \\ Received: 12 November 2017 / Revised: 6 October 2018 / Published online: 4 April 2019 \\ (c) The Author(s) 2019
}

\begin{abstract}
By using analytic tools from stochastic analysis, we initiate a study of some non-linear parabolic equations on Sierpinski gasket, motivated by modellings of fluid flows along fractals (which can be considered as models of simplified rough porous media). Unlike the regular space case, such parabolic type equations involving non-linear convection terms must take a different form, due to the fact that convection terms must be singular to the "linear part" which defines the heat semigroup. In order to study these parabolic type equations, a new kind of Sobolev inequalities for the Dirichlet form on the gasket will be established. These Sobolev inequalities, which are interesting on their own and in contrast to the case of Euclidean spaces, involve two $L^{p}$ norms with respect to two mutually singular measures. By examining properties of singular convolutions of the associated heat semigroup, we derive the space-time regularity of solutions to these parabolic equations under a few technical conditions. The Burgers equations on the Sierpinski gasket are also studied, for which a maximum principle for solutions is derived using techniques from backward stochastic differential equations, and the existence, uniqueness, and regularity of its solutions are obtained.
\end{abstract}

Keywords Brownian motion · Dirichlet forms · Sierpinski gasket · Sobolev inequalities $\cdot$ Semi-linear parabolic equations

Mathematics Subject Classification $28 \mathrm{~A} 80 \cdot 60 \mathrm{~J} 45$

X. Liu: This research was carried out when this author was reading DPhil in Mathematics at the University of Oxford.

Z. Qian: Research supported partly by an ERC grant (Grant Agreement No. 291244 ESig).

Zhongmin Qian

zhongmin.qian@maths.ox.ac.uk

Xuan Liu

chamonixliu@163.com

1 Nomura International, 30/FL Two International Finance Centre, Hong Kong, Hong Kong

2 Mathematical Institute, University of Oxford, Oxford OX2 6GG, UK 


\section{Introduction}

The analysis on fractals has attracted attentions of researchers in the last decades, not only for the reason that fractals are archetypal examples of spaces without suitable smooth structure, but also because fractals are examples of interesting models in statistical mechanics. Many objects in nature (e.g. percolation clusters in disordered media, complex biology systems, polymeric materials, and etc.) possess features of fractals (see e.g. [28] for details). Fractals appear as scaling limits of lattices. Lattice models (e.g. the Ising models and their variants) have been extensively studied in statistical mechanics, and properties for scaling limits have been derived using conformal field theory in dimension two.

Since a calculus on fractals is not available, the theory of Dirichlet forms on measure-metric spaces and stochastic calculus are the analytic tools employed for the study of analysis problems on fractals, and many interesting results have been established in the past decades.

Early works on analysis on fractals however have been focused mainly on diffusion processes and the corresponding Dirichlet forms (see e.g. [1-3,8,11,12,21$24]$ and etc.). Brownian motion on the Sierpinski gasket was first constructed by Goldstein and Kusuoka as the limit of a sequence of (scaled) random walks on lattices (cf. [9,26]). Kigami [22] has obtained an analytic construction of the Dirichlet form via finite difference schemes. The construction of gradients of functions with finite energy has been given in Kusuoka in [25], where a significant difference between Euclidean spaces and fractals has also been revealed (see [25, Section 6]). On the Sierpinski gasket for example, volumes of sets and energies of functions are measured in terms of two mutually singular measures, the Hausdorff measure and Kusuoka's measure (see Sect. 2 below for definitions). By virtue of the results obtained in [25], gradients of functions on the Sierpinski gasket may be defined as square integrable functions with respect to Kusuoka's measure (cf. Sect. 2). Roughly speaking, the gradient of a function with finite energy is the square root of the density of its energy measure with respect to Kusuoka's measure. There have been interests in the understanding of gradients of functions and non-linear partial differential equations on fractals with nonlinearities involving first-order derivatives (see e.g. [16,18-20,33] and references therein). A new class of semi-linear parabolic equations involving singular measures on the Sierpinski gasket was proposed and studied in [27], where, among other things, a Feynman-Kac representation was obtained assuming the existence of weak solutions.

In the present paper, we establish the existence and uniqueness of solutions to the semi-linear parabolic PDEs proposed in [27], and derive the regularity of solutions. A crucial ingredient in our argument is a new type of Sobolev inequalities on the Sierpinski gasket (and the infinite gasket) involving different measures (which can be mutually singular). To author's knowledge, this type of Sobolev inequalities on fractals has not been investigated before, and is of mathematical interests on its own. We formulate and study the Burgers equations on the gasket, which is an archetype of non-linear PDEs with non-Lipschitz coefficients, and also as a simplified model of flows in porous medium. The difficulty in our case is that there 
exists no suitable analogue of the Cole-Hopf transformation on the gasket. Instead we tackle the problem by using a Feynman-Kac representation and an iteration argument.

This paper is organized as follows. We introduce in Sect. 2 the notations and definitions which will be effective throughout the paper. Several preliminary results are also reviewed in the same section. In Sect. 3, we give the formulation and the proof of new Sobolev inequalities on the Sierpinski gasket (and the infinite gasket), which will be needed in latter sections. The optimal exponents and a sufficient and necessary condition for the validity of these inequalities are also given in this section. Section 4 is devoted to the semi-linear parabolic PDEs on the gasket, where we establish the existence and uniqueness and the regularity of solutions. In Sect. 5, we apply the results in previous sections to the study of the Burgers equations on the gasket, which are the analogues of the Burgers equations on $\mathbb{R}$.

The results of this paper are presented only for the Sierpinski gasket in $\mathbb{R}^{2}$, we however believe that our results also hold for Sierpinski gaskets in higher dimensions. The main results and the arguments given in this paper can be adapted accordingly without difficulties.

\section{Preliminaries}

In this section, we set up several notations and definitions which will be in force throughout this paper.

\section{Sierpinski gaskets}

Let $\mathbf{F}_{i}: \mathbb{R}^{2} \rightarrow \mathbb{R}^{2}, i=1,2,3$ be the contractions defined by $\mathbf{F}_{1}(x)=2^{-1} x, \mathbf{F}_{2}(x)$ $=2^{-1}[x+(1,0)], \quad \mathbf{F}_{3}(x)=2^{-1}[x+(1 / 2, \sqrt{3} / 2)], \quad x \in \mathbb{R}^{2}$. Let $\mathrm{V}_{0}=\{(0,0)$, $(1,0),(1 / 2, \sqrt{3} / 2)\}$. Define $\mathrm{V}_{m}, m \in \mathbb{N}_{+}$inductively by $\mathrm{V}_{m}=\bigcup_{i=1,2,3} \mathbf{F}_{i}\left(\mathrm{~V}_{m-1}\right)$. Let $\hat{\mathrm{V}}_{m}=\bigcup_{k=0}^{\infty} 2^{k}\left[\mathrm{~V}_{m+k} \cup\left(-\mathrm{V}_{m+k}\right)\right], m \in \mathbb{N}$. The (compact) Sierpinski gasket $\mathbb{S}$ and the infinite Sierpinski gasket $\hat{\mathbb{S}}$ are defined to be the closures $\mathbb{S}=\operatorname{cl}\left(\bigcup_{m=0}^{\infty} \mathrm{V}_{m}\right)$ and $\hat{\mathbb{S}}=\operatorname{cl}\left(\bigcup_{m=0}^{\infty} \hat{\mathrm{V}}_{m}\right)$ respectively. $\hat{\mathbb{S}}$ can be written as a countable union $\hat{\mathbb{S}}=$ $\bigcup_{i \in \mathbb{Z}} \tau_{i}(\mathbb{S})$, where $\tau_{i}: \mathbb{R}^{2} \rightarrow \mathbb{R}^{2}, i \in \mathbb{Z}$ are translations of $\mathbb{R}^{2}$ such that $\tau_{i}(\mathbb{S}), i \in \mathbb{Z}$ have non-overlapping interiors. To our purpose, the labelling of the translations $\tau_{i}$, $i \in \mathbb{Z}$ is immaterial. We should point out that there are many different infinite versions of $\mathbb{S}$ (see e.g. [32, Section 5]). The $\hat{\mathbb{S}}$ we use in the present paper is only one of them.

Let $\mathrm{W}_{*}=\left\{\omega=\omega_{1} \omega_{2} \omega_{3} \ldots: \omega_{i} \in\{1,2,3\}, i \in \mathbb{N}_{+}\right\}$the set of infinite ordered sequences $\omega$ of symbols in $\{1,2,3\}$. For each $\omega=\omega_{1} \omega_{2} \omega_{3} \ldots \in \mathrm{W}_{*}$ and each $m \in \mathbb{N}_{+}$, let $[\omega]_{m}=\omega_{1} \omega_{2} \ldots \omega_{m}$, define $\mathbf{F}_{[\omega]_{m}}=\mathbf{F}_{\omega_{1}} \mathbf{F}_{\omega_{2}} \cdots \mathbf{F}_{\omega_{m}}$, and $\mathbb{S}_{[\omega]_{m}}=\mathbf{F}_{[\omega]_{m}}(\mathbb{S})$. As a convention, we define $\mathbf{F}_{[\omega]_{0}}=\mathbf{I d}$. The Hausdorff measure on $\mathbb{S}$ is the unique Borel probability measure $v$ on $\mathbb{S}$ such that $\nu\left(\mathbb{S}_{[\omega]_{m}}\right)=3^{-m}$ for all $\omega \in \mathrm{W}_{*}, m \in \mathbb{N}$, and the Hausdorff measure on $\hat{\mathbb{S}}$ is the unique Borel measure $\hat{v}$ on $\hat{\mathbb{S}}$ such that $\left.\left(\hat{v} \circ \tau_{i}\right)\right|_{\mathbb{S}}=v$ for all $i \in \mathbb{Z}$. 


\section{Standard Dirichlet forms}

For each $m \in \mathbb{N}$ and any functions $u, v$ on $\bigcup_{m=0}^{\infty} \mathrm{V}_{m}$, let

$$
\mathcal{E}^{(m)}(u, v)=\sum_{x, y \in \mathrm{V}_{m}:|x-y|=2^{-m}} 2^{-1}(5 / 3)^{m}[u(x)-u(y)][v(x)-v(y)] .
$$

The sequence $\left\{\mathcal{E}^{(m)}(u, u)\right\}_{m \in \mathbb{N}}$ is non-decreasing (cf. [24, Sections 2.2, 2.4]), therefore $\mathcal{E}(u, u)=\lim _{m \rightarrow \infty} \mathcal{E}^{(m)}(u, u)$ exists (possibly infinite), and the limit will be denoted by $\mathcal{E}(u)$ for simplicity.

Let $\mathcal{F}(\mathbb{S})=\left\{u: u\right.$ is a function on $\bigcup_{m=0}^{\infty} \mathrm{V}_{m}$ with $\left.\mathcal{E}(u)<\infty\right\}$. According to [24, Theorem 2.2.6], every function $u \in \mathcal{F}(\mathbb{S})$ uniquely extends to a continuous function on $\mathbb{S}$, in other words, $\mathcal{F}(\mathbb{S}) \subseteq C(\mathbb{S})$. $(\mathcal{E}, \mathcal{F}(\mathbb{S}))$ is called the standard Dirichlet form on $\mathbb{S}$, which is a regular local Dirichlet form on $L^{2}(\mathbb{S} ; v)$. $(\mathcal{E}, \mathcal{F}(\mathbb{S}))$ possesses the property of self-similarity in the sense that

$$
\mathcal{E}(u, v)=\sum_{i=1,2,3}(5 / 3) \mathcal{E}\left(u \circ \mathbf{F}_{i}, v \circ \mathbf{F}_{i}\right), \quad u, v \in \mathcal{F}(\mathbb{S})
$$

Let $\mathcal{L}$ be the self-adjoint non-positive operator on $\operatorname{Dom}(\mathcal{L}) \subseteq L^{2}(\mathbb{S} ; v)$ associated with $(\mathcal{E}, \mathcal{F}(\mathbb{S}))$.

Let $\mathcal{F}\left(\mathbb{S} \backslash \mathrm{V}_{0}\right)=\left\{u \in \mathcal{F}(\mathbb{S}):\left.u\right|_{\mathrm{V}_{0}}=0\right\}$. The restricted form $\left(\mathcal{E}, \mathcal{F}\left(\mathbb{S} \backslash \mathrm{V}_{0}\right)\right)$ is also a regular local Dirichlet form on $L^{2}(\mathbb{S} ; v)$ corresponding to Dirichlet boundary conditions.

By replacing $\mathrm{V}_{m}$ with $\hat{\mathrm{V}}_{m}$ in $(2.1), \hat{\mathcal{E}}(u)$ can be defined similarly for any $u \in C(\hat{\mathbb{S}})$. Let $\mathcal{F}(\widehat{\mathbb{S}})$ be the completion of $\{u \in C(\hat{\mathbb{S}}): \hat{\mathcal{E}}(u)<\infty\}$ with respect to the norm $\hat{\mathcal{E}}(\cdot)^{1 / 2}+\|\cdot\|_{L^{2}(\hat{v})}$. It can be shown that $\mathcal{F}(\hat{\mathbb{S}}) \subseteq C_{0}(\hat{\mathbb{S}})$, where $C_{0}(\hat{\mathbb{S}})$ is the space of continuous functions on $\hat{\mathbb{S}}$ vanishing at infinity. $(\hat{\mathcal{E}}, \mathcal{F}(\hat{\mathbb{S}}))$ is called the standard Dirichlet form on $\hat{\mathbb{S}}$, which is a regular local Dirichlet form on $L^{2}(\hat{\mathbb{S}} ; \hat{v})$. By definition

$$
\hat{\mathcal{E}}(u, v)=\sum_{i \in \mathbb{Z}} \mathcal{E}\left[\left.\left(u \circ \tau_{i}\right)\right|_{\mathbb{S}},\left.\left(v \circ \tau_{i}\right)\right|_{\mathbb{S}}\right], \quad u, v \in \mathcal{F}(\hat{\mathbb{S}})
$$

Similar to $\mathcal{E}$, the form $\hat{\mathcal{E}}$ is self-similar in the sense that

$$
\hat{\mathcal{E}}(u, v)=(5 / 3) \hat{\mathcal{E}}\left(u \circ \mathbf{F}_{1}, v \circ \mathbf{F}_{1}\right), \quad u, v \in \mathcal{F}(\hat{\mathbb{S}}) .
$$

For any $x, y \in \hat{\mathbb{S}}$, define $R(x, y)$ by

$$
R(x, y)^{-1}=\inf \{\hat{\mathcal{E}}(u): u \in \mathcal{F}(\hat{\mathbb{S}}), u(x)=0, u(y)=1\}
$$

if $x \neq y$, and $R(x, y)=0$ if $x=y$. For every $x, y \in \hat{\mathbb{S}}, R(x, y)<\infty$. Moreover, if $x \neq y$, then there exists a unique $u \in \mathcal{F}(\hat{\mathbb{S}})$ such that $u(x)=1, u(y)=0, \hat{\mathcal{E}}(u)=$ 
$R(x, y)^{-1}$ (see [24, Theorem 2.3.4]). The function $R(\cdot, \cdot)$, called the resistance metric, is a metric on $\hat{\mathbb{S}}$ satisfying

$$
C_{*}^{-1}|x-y|^{d_{w}-d_{f}} \leq R(x, y) \leq C_{*}|x-y|^{d_{w}-d_{f}}, x, y \in \hat{\mathbb{S}}
$$

for some universal constant $C_{*} \geq 1$, where $d_{s}=2 \log 3 / \log 5, d_{w}=\log 5 / \log 2$, and $d_{f}=d_{w} /\left(2 d_{s}\right)$ are the spectral dimension, the walk dimension, and the fractal dimension of $\hat{\mathbb{S}}$ respectively (cf. [24, Lemma 3.3.5]). By the definition of $R(\cdot, \cdot)$,

$$
|u(x)-u(y)| \leq R(x, y)^{1 / 2} \hat{\mathcal{E}}(u)^{1 / 2}, \quad u \in \mathcal{F}(\hat{\mathbb{S}}), x, y \in \hat{\mathbb{S}}
$$

Since $\left.u\right|_{\mathbb{S}} \in \mathcal{F}(\mathbb{S}), u \in \mathcal{F}(\hat{\mathbb{S}})$ and $\max _{\mathbb{S} \times \mathbb{S}} R<\infty$, it follows from (2.4) that

$$
\underset{\mathbb{S}}{\operatorname{osc}}(u) \leq C_{*} \mathcal{E}(u)^{1 / 2}, \quad u \in \mathcal{F}(\mathbb{S}) .
$$

Let $f$ be a function on $\mathrm{V}_{0}$. There exists a unique $h \in \mathcal{F}(\mathbb{S})$ such that $\left.h\right|_{\mathrm{v}_{0}}=f$ and $\mathcal{E}(h)=\inf \left\{\mathcal{E}(u): u \in \mathcal{F}(\mathbb{S}),\left.u\right|_{\mathrm{v}_{0}}=f\right\}$ (see [24, Proposition 3.2.1]). $h$ is called the harmonic function in $\mathbb{S}$ with boundary value $\left.h\right|_{\mathrm{v}_{0}}=f$. A harmonic function $h$, restricted on each $\mathrm{V}_{m}, m \in \mathbb{N}$, can be evaluated by

$$
\left.\left(h \circ \mathbf{F}_{[\omega]_{m}}\right)\right|_{\mathrm{v}_{0}}=\mathbf{A}_{[\omega]_{m}}\left(\left.h\right|_{\mathrm{v}_{0}}\right), \quad \omega=\omega_{1} \omega_{2} \omega_{3} \ldots \in \mathrm{W}_{*},
$$

(cf. [24, Proposition 3.2.1 ]), where $\mathbf{A}_{[\omega]_{m}}=\mathbf{A}_{\omega_{m}} \cdots \mathbf{A}_{\omega_{2}} \mathbf{A}_{\omega_{1}}$, with

$$
\mathbf{A}_{1}=\frac{1}{5}\left[\begin{array}{lll}
5 & 0 & 0 \\
2 & 2 & 1 \\
2 & 1 & 2
\end{array}\right], \mathbf{A}_{2}=\frac{1}{5}\left[\begin{array}{lll}
2 & 2 & 1 \\
0 & 5 & 0 \\
1 & 2 & 2
\end{array}\right], \mathbf{A}_{3}=\frac{1}{5}\left[\begin{array}{lll}
2 & 1 & 2 \\
1 & 2 & 2 \\
0 & 0 & 5
\end{array}\right] .
$$

Let $f$ be a function on $\mathrm{V}_{m}$. The m-harmonic function with boundary value $f$ is defined to be the unique $h \in \mathcal{F}(\mathbb{S})$ such that $\left.h\right|_{\mathrm{V}_{m}}=f$ and that $h \circ \mathbf{F}_{[\omega]_{m}}$ is a harmonic function for all $\omega \in \mathrm{W}_{*}$. The energy of an $m$-harmonic function $h$ can be calculated using $\mathcal{E}(h)=\mathcal{E}^{(m)}\left(\left.h\right|_{\mathrm{V}_{m}}\right)$.

\section{Kusuoka measures and gradients}

Let $\mathbf{P}: \mathbb{R}^{3} \rightarrow \mathbb{R}^{3}$ be the projection $\mathbf{P} x=x-\left(x_{1}+x_{2}+x_{3}\right) / 3$ for $x=\left(x_{1}, x_{2}, x_{3}\right) \in$ $\mathbb{R}^{3}$. The Kusuoka measure $\mu$ on $\mathbb{S}$, as defined in [25], is the unique Borel probability measure on $\mathbb{S}$ such that

$$
\mu\left(\mathbb{S}_{[\omega]_{m}}\right)=2^{-1} \cdot(5 / 3)^{m} \operatorname{tr}\left(\mathbf{A}_{[\omega]_{m}}^{\mathrm{t}} \mathbf{P} \mathbf{A}_{[\omega]_{m}}\right)
$$

for all $\omega=\omega_{1} \omega_{2} \omega_{3} \ldots \in \mathrm{W}_{*}, m \in \mathbb{N}$. The Kusuoka measure $\hat{\mu}$ on $\hat{\mathbb{S}}$ is the unique Borel measure on $\widehat{\mathbb{S}}$ such that $\left.\left(\hat{\mu} \circ \tau_{i}\right)\right|_{\mathbb{S}}=\mu$ for all $i \in \mathbb{Z}$.

The Kusuoka measure $\mu$ ( $\hat{\mu}$ respectively) is singular to the Hausdorff measure $v$ ( $\hat{v}$ respectively) (cf. [25, p. 678]). If $u \in \mathcal{F}(\mathbb{S})$, then $\mu_{\langle u\rangle}$ denotes the energy measure 
of $u$, i.e. the Borel measure on $\mathbb{S}$ such that $\int_{\mathbb{S}} \phi d \mu\langle u\rangle=\mathcal{E}(\phi u, u)-2^{-1} \mathcal{E}\left(\phi, u^{2}\right)$ for $\phi \in \mathcal{F}(\mathbb{S})$. By [25, Theorem (5.4)], $\mu\langle u\rangle \ll \mu$ for all $u \in \mathcal{F}(\mathbb{S})$ (see [13,15] for similar results on general fractals). Moreover, there exists a unique linear operator $\nabla: \mathcal{F}(\mathbb{S}) \rightarrow L^{2}(\mu)$, called the gradient operator on $\mathbb{S}$, satisfying the following: (i) $\mu_{\langle u\rangle}=|\nabla u|^{2} \mu$ for all $u \in \mathcal{F}(\mathbb{S})$, and (ii) if $h$ is the harmonic function with boundary value $h(0,0)=0, h(1,0)=h(1 / 2, \sqrt{3} / 2)=1$, then $\nabla h>0 \quad \mu$-a.e. The gradient operator $\nabla: \mathcal{F}(\hat{\mathbb{S}}) \rightarrow L^{2}(\hat{\mu})$ on $\hat{\mathbb{S}}$ is defined by $\left.\left[(\nabla u) \circ \tau_{i}\right]\right|_{\mathbb{S}}=\nabla\left[\left.\left(u \circ \tau_{i}\right)\right|_{\mathbb{S}}\right]$ for all $u \in \mathcal{F}(\hat{\mathbb{S}})$ and all $i \in \mathbb{Z}$.

Remark 2.1 We should point out that there exist several slight variants of gradients on fractals, which are introduced to address different problems (see, e. g. [4,6,14,23,27, 30,33 ] and references therein). The definition of gradients on $\mathbb{S}$ adopted in the present paper was introduced in [27] via martingale representations, and can be regarded as the special case of the definition given in [14], where $\mu$ is the minimal energy-dominant measure (see [14, p. 3] for the definition).

\section{Sobolev inequalities}

The objective of this section is to establish some Sobolev inequalities involving different (probably mutually singular) measures on $\mathbb{S}$ and $\hat{\mathbb{S}}$ (Theorems 3.6, 3.11 respectively), which is crucial to our study of some semi-linear parabolic equations on the gasket. A sufficient and necessary condition for the validity of these Sobolev inequalities (Theorems 3.8, 3.13) will be established as well.

To shed some light on the motivation of these inequalities, consider the following simple parabolic PDE on $\mathbb{S}$

$$
\partial_{t} u d v=\mathcal{L} u d v+\nabla u d \mu .
$$

Here the singular measures $v$ and $\mu$ must be involved as $\mathcal{L} u$ is $v$-a.e. defined while $\nabla u$ is only $\mu$-a.e. defined. A precise interpretation of this equation will be given in Sect. 4 . Let us assume for the moment that if $u$ is a solution then one may test the equation against the solution to obtain

$$
\frac{d}{d t}\|u(t)\|_{L^{2}(v)}^{2}=-\mathcal{E}(u(t))+\langle\nabla u(t), u(t)\rangle_{\mu},
$$

from which it follows that

$$
\frac{d}{d t}\|u(t)\|_{L^{2}(v)}^{2} \leq-\frac{1}{2} \mathcal{E}(u(t))+\frac{1}{2}\|u(t)\|_{L^{2}(\mu)}^{2} .
$$

For PDEs on Euclidean spaces, the measures $v$ and $\mu$ are equal to the Lebesgue measures, and therefore, the above differential inequality together with Grönwall's inequality yields the energy estimates and the existence and uniqueness of solutions. However, on $\mathbb{S}$, the measures $v$ and $\mu$ are mutually singular, and hence the $L^{2}$-norms $\|\cdot\|_{L^{2}(v)}$ and $\|\cdot\|_{L^{2}(\mu)}$ are in general incomparable. Thus, Grönwall's inequality does 
not apply in this case. For PDEs involving gradients on $\mathbb{S}$, an appropriate comparison of $\|\cdot\|_{L^{2}(v)}$ and $\|\cdot\|_{L^{2}(\mu)}$ is necessary to obtaining energy estimates. In fact, for functions $u \in \mathcal{F}(\mathbb{S})$, the $L^{2}$-norms $\|u\|_{L^{2}(v)}$ and $\|u\|_{L^{2}(\mu)}$ must be compared with the involvement of (an arbitrarily small portion of) the energy $\mathcal{E}(u)$ (see Corollary 3.18 below). This type of comparison is possible due to the Sobolev inequalities to be established in this section.

For convenience, $C_{*}$ will always denote a generic universal constant which may be different on various occasions.

Definition 3.1 Let $S_{i, m}=2^{m} \tau_{i}(\mathbb{S}), m, i \in \mathbb{Z}$. The energy of $u \in \mathcal{F}(\hat{\mathbb{S}})$ on $S_{i, m}$ is defined to be $\left.\hat{\mathcal{E}}\right|_{S_{i, m}}(u)=(3 / 5)^{m} \mathcal{E}\left[\left.\left(u \circ \tau_{i} \circ \mathbf{F}_{1}^{-m}\right)\right|_{\mathbb{S}}\right]$.

Clearly, $\hat{\mathbb{S}}$ can be written as the non-overlapping union $\hat{\mathbb{S}}=\bigcup_{i \in \mathbb{Z}} S_{i, m}$ for each $m \in \mathbb{Z}$. Therefore, $\hat{\mathcal{E}}(u)=\left.\sum_{i \in \mathbb{Z}} \hat{\mathcal{E}}\right|_{S_{i, m}}(u)$ for any $u \in \mathcal{F}(\hat{\mathbb{S}})$ in view of (2.2) and (2.3).

Definition 3.2 The constant $\delta_{s}>0$ is defined by $1 / \delta_{s}=2 / d_{s}-1=\log 5 / \log 3-1$.

The constant $\delta_{s}$ is defined so that $5 / 3=3^{1 / \delta_{s}}$. Therefore, for every $i$ and $m$, by (2.5),

$$
\begin{aligned}
& \underset{\mathbb{S}}{\operatorname{osc}}\left(u \circ \tau_{i} \circ \mathbf{F}_{1}^{-m}\right) \leq C_{*} \mathcal{E}\left[\left(u \circ \tau_{i} \circ \mathbf{F}_{1}^{-m}\right) \mid \mathbb{S}\right]^{1 / 2} \\
& \quad=\left.C_{*}(5 / 3)^{m / 2} \hat{\mathcal{E}}\right|_{S_{i, m}}(u)^{1 / 2}=\left.C_{*} \hat{v}\left(S_{i, m}\right)^{1 /\left(2 \delta_{s}\right)} \hat{\mathcal{E}}\right|_{S_{i, m}}(u)^{1 / 2},
\end{aligned}
$$

which implies that

$$
\underset{S_{i, m}}{\operatorname{OSc}}(u) \leq\left. C \hat{v}\left(S_{i, m}\right)^{1 /\left(2 \delta_{s}\right)} \hat{\mathcal{E}}\right|_{S_{i, m}}(u)^{1 / 2}
$$

Definition 3.3 A subset $S \subseteq \hat{\mathbb{S}}$ is called a dyadic triangle if $S=S_{i, m}$ for some $m, i \in \mathbb{Z}$.

We are now in a position to formulate the main results of this section. Let $\hat{\sigma}$ be a Borel measure on $\hat{\mathbb{S}}$ satisfying the following condition: there exist constants $C_{\hat{\sigma}} \geq 1$ and $0<\underline{\delta} \leq \bar{\delta} \leq \infty, \bar{\delta} \geq 1$ such that

$$
\begin{cases}\hat{\sigma}(S) \leq C_{\hat{\sigma}} \hat{v}(S)^{1 / \bar{\delta}}, & \text { if } 0<\operatorname{diam}(S)<1 \\ \hat{\sigma}(S) \leq C_{\hat{\sigma}} \hat{v}(S)^{1 / \underline{\delta}}, & \text { if } \operatorname{diam}(S) \geq 1\end{cases}
$$

for any dyadic triangle $S \subseteq \widehat{\mathbb{S}}$, where $\operatorname{diam}(A)$ denotes the diameter of $A \subseteq \widehat{\mathbb{S}}$ with respect to the Euclidean metric.

Remark 3.4 (i) In literature, a Borel measure $\sigma$ on $\mathbb{R}^{n}$ is called an Ahlfors regular measure if there exists a $d>0$ such that $C^{-1} r^{d} \leq \sigma(B(x, r)) \leq C r^{d}$ for any ball of radius $r$ centred at $x \in \mathbb{R}^{n}$. Therefore, the Hausdorff measure is an Ahlfors regular measure, and we think it is appropriate to call the measure $\hat{\sigma}$ in (M.1) an Ahlfors upper regular measure (with distinct exponents for expansion and 
contraction). In the present paper, we formulate the condition (M.1) in terms of the Hausdorff measure rather than diameter of sets because of notational convenience when comparing measures. We would like to mention that a heat kernel estimate implies the Ahlfors regularity of the Hausdorff measure (see e.g. [10, Theorem 3.2] and references therein).

(ii) The restriction $\bar{\delta} \geq 1$ in the condition (M.1) is necessary in view of the countable additivity of measures.

(iii) Notice that we do not require (M.1) to hold for general Borel sets. In fact, (M.1) being valid for all Borel sets implies the absolute continuity of $\hat{\sigma}$ with respect to $\hat{v}$.

We would like to point out that the condition (M.1) is general enough to include many cases of interests, some of important examples are listed below.

Example 3.5 (i) Dirac measures, for which the condition (M.1) is satisfied with $\underline{\delta}=$ $\bar{\delta}=\infty$.

(ii) The Kusuoka measure $\hat{\mu}$ (cf. Corollary 3.9.(b) below).

(iii) Analogues on $\hat{\mathbb{S}}$ of weighted measures $|x|^{-\theta} d x$ on $\mathbb{R}^{d}$ with $0 \leq \theta<d$. For any cube $Q \subseteq \mathbb{R}^{d}$, we have $\int_{Q}|x|^{-\theta} d x \leq C|Q|^{1-\theta / d}$ for some constant $C>0$ depending only on $d$. Therefore, the analogue on $\hat{\mathbb{S}}$ of $|x|^{-\theta} d x$ on $\mathbb{R}^{d}$ would be a Borel measure $\hat{\sigma} \ll \hat{v}$ satisfying the condition (M.1) with $\underline{\delta}, \bar{\delta}$ given by $1 / \underline{\delta}=1 / \bar{\delta}=1-\theta / d_{s}$. Here we have used $d_{s}$ as the Sobolev dimension of $\hat{\mathbb{S}}$ (cf. Remark 3.7 below).

Theorem 3.6 Let $1 \leq p \leq q \leq \infty, q \geq 2$. Suppose $\hat{\sigma}$ is a Borel measure on $\hat{\mathbb{S}}$ satisfying the condition (M.1). Then

$$
\|u\|_{L^{q}(\hat{\sigma})} \leq C \sum_{i=1,2} \hat{\mathcal{E}}(u)^{a_{i} / 2}\|u\|_{L^{p}(\hat{v})}^{1-a_{i}}, \quad u \in \mathcal{F}(\hat{\mathbb{S}}),
$$

where

$$
a_{1}=\left[\frac{1 / p-1 /(q \bar{\delta})}{1 / p+1 /\left(2 \delta_{s}\right)}\right]^{+}, a_{2}=\left[\frac{1 / p-1 /(q \underline{\delta})}{1 / p+1 /\left(2 \delta_{s}\right)}\right]^{+},
$$

and $C>0$ is a constant depending only on the constant $C_{\hat{\sigma}}$ in (M.1).

Moreover, if there exists a sequence $\left\{S_{m}\right\}_{m \in \mathbb{Z}}$ of dyadic triangles such that

$$
\lim _{m \rightarrow-\infty} \operatorname{diam}\left(S_{m}\right)=0, \quad \lim _{m \rightarrow \infty} \operatorname{diam}\left(S_{m}\right)=\infty
$$

and

$$
1 / \bar{\delta}=\lim _{m \rightarrow-\infty} \frac{\log \hat{\sigma}\left(S_{m}\right)}{\log \hat{v}\left(S_{m}\right)}, 1 / \underline{\delta}=\lim _{m \rightarrow \infty} \frac{\log \hat{\sigma}\left(S_{m}\right)}{\log \hat{v}\left(S_{m}\right)},
$$

then the pair of exponents given by (3.3) is optimal in the following sense: if

$$
\|u\|_{L^{q}(\hat{\sigma})} \leq C \sum_{i=1}^{N} \hat{\mathcal{E}}(u)^{b_{i} / 2}\|u\|_{L^{p}(\hat{v})}^{1-b_{i}}, \quad u \in \mathcal{F}(\hat{\mathbb{S}}),
$$


for some constants $b_{i} \in[0,1], 1 \leq i \leq N, N \in \mathbb{N}_{+}$and $C>0$ independent of $u$, then $\min _{i} b_{i} \leq a_{2} \leq a_{1} \leq \max _{i} b_{i}$.

Proof Suppose first that $p \leq q<\infty$. Let $\hat{v}_{m}=\hat{v}\left(2^{m} \mathbb{S}\right)=3^{m}, S_{i, m}=2^{m} \tau_{i}(\mathbb{S})$ for any $m, i \in \mathbb{Z}$. Then $\hat{\mathbb{S}}=\bigcup_{i} S_{i, m}$. When $m \geq 0$, by (3.1), we have that

$$
\begin{aligned}
& \int_{\hat{\mathbb{S}}}|u|^{q} d \hat{\sigma} \leq 2^{q-1} \sum_{i}\left[\int_{S_{i, m}}\left|u-\frac{1}{\hat{v}_{m}} \int_{S_{i, m}} u d \hat{v}\right|^{q} d \hat{\sigma}+\left|\frac{1}{\hat{v}_{m}} \int_{S_{i, m}} u d \hat{v}\right|^{q} \hat{\sigma}\left(S_{i, m}\right)\right] \\
& \leq C^{q} \sum_{i}\left[\left.\hat{v}_{m}^{q /\left(2 \delta_{s}\right)} \hat{\mathcal{E}}\right|_{S_{i, m}}(u)^{q / 2} \hat{\sigma}\left(S_{i, m}\right)+\frac{1}{\hat{v}_{m}^{q / p}}\left[\int_{S_{i, m}}|u|^{p} d \hat{v}\right]^{q / p} \hat{\sigma}\left(S_{i, m}\right)\right] \\
& \leq C^{q} \sum_{i}\left[\left.\hat{v}_{m}^{q /\left(2 \delta_{s}\right)+1 / \underline{\delta}} \hat{\mathcal{E}}\right|_{S_{i, m}}(u)^{q / 2}+\hat{v}_{m}^{1 / \underline{\delta}-q / p}\left(\int_{S_{i, m}}|u|^{p} d \hat{v}\right)^{q / p}\right] \\
& \leq C^{q}\left\{\hat{v}_{m}^{q /\left(2 \delta_{s}\right)+1 / \underline{\delta}}\left[\left.\sum_{i} \hat{\mathcal{E}}\right|_{S_{i, m}}(u)\right]^{q / 2}+C^{q} \hat{v}_{m}^{1 / \underline{\delta}-q / p}\left[\sum_{i} \int_{S_{i, m}}|u|^{p} d \hat{v}\right]^{q / p}\right\} \\
& =C^{q}\left[\hat{v}_{m}^{q /\left(2 \delta_{s}\right)+1 / \underline{\delta}} \hat{\mathcal{E}}(u)^{q / 2}+\hat{v}_{m}^{1 / \underline{\delta}-q / p}\|u\|_{L^{p}(\hat{v})}^{q}\right] \text {, }
\end{aligned}
$$

where and hereafter $C>0$ denotes a generic constant depending only on the constant $C_{\hat{\sigma}}$ in (M.1). Therefore,

$$
\|u\|_{L^{q}(\hat{\sigma})} \leq C\left[\hat{v}_{m}^{1 /\left(2 \delta_{s}\right)+1 /(q \underline{\delta})} \hat{\mathcal{E}}(u)^{1 / 2}+\hat{v}_{m}^{1 /(q \underline{\delta})-1 / p}\|u\|_{L^{p}(\hat{v})}\right]
$$

Similarly, when $m \leq 0$, we have that

$$
\|u\|_{L^{q}(\hat{\sigma})} \leq C\left[\hat{v}_{m}^{1 /\left(2 \delta_{s}\right)+1 /(q \bar{\delta})} \hat{\mathcal{E}}(u)^{1 / 2}+\hat{v}_{m}^{1 /(q \bar{\delta})-1 / p}\|u\|_{L^{p}(\hat{v})}\right]
$$

The proof of (3.2) is done by optimising over $m$. Suppose $\hat{\mathcal{E}}(u)^{1 / 2} \geq\|u\|_{L^{p}(\hat{v})}$. Consider the following two cases:

Case 1: $p \leq q \leq p / \bar{\delta}$. Note that $p \leq p / \bar{\delta}$ forces $\bar{\delta}=1$ and therefore $1 /(q \bar{\delta})=$ $1 / p, a_{1}=0$. Setting $m \rightarrow-\infty$ in (3.6) gives that

$$
\|u\|_{L^{q}(\hat{\sigma})} \leq C \hat{\mathcal{E}}(u)^{a_{1} / 2}\|u\|_{L^{p}(\hat{v})}^{1-a_{1}} .
$$

Case 2: $q>p / \bar{\delta}$. Setting

$$
m=\sup \left\{m \leq 0: \hat{v}_{m}^{1 /\left(2 \delta_{s}\right)+1 / p} \leq \hat{\mathcal{E}}(u)^{-1 / 2}\|u\|_{L^{p}(\hat{v})} \leq 1\right\}<\infty,
$$

in (3.6), we obtain that

$$
\|u\|_{L^{q}(\hat{\sigma})} \leq C \hat{\mathcal{E}}(u)^{a_{1} / 2}\|u\|_{L^{p}(\hat{v})}^{1-a_{1}}, \text { where } a_{1}=\left[\frac{1 / p-1 /(q \bar{\delta})}{1 / p+1 /\left(2 \delta_{s}\right)}\right]^{+} .
$$

Suppose that $\hat{\mathcal{E}}(u)^{1 / 2}<\|u\|_{L^{p}(\hat{v})}$. We consider the two cases. 
Case 1: $p \leq q \leq p / \underline{\delta}$. In this case, $a_{2}=0$. Setting $m=0$ in (3.5) gives that

$$
\|u\|_{L^{q}(\hat{\mu})} \leq C \hat{\mathcal{E}}(u)^{a_{2} / 2}\|u\|_{L^{p}(\hat{v})}^{1-a_{2}} .
$$

Case $2: q>p / \underline{\delta}$. Setting

$$
m=\inf \left\{m \geq 0: \hat{v}_{m}^{1 /\left(2 \delta_{s}\right)+1 / p} \geq \hat{\mathcal{E}}(u)^{-1 / 2}\|u\|_{L^{p}(\hat{v})}>1\right\}<\infty
$$

in (3.5), we obtain that

$$
\|u\|_{L^{q}(\hat{\sigma})} \leq C \hat{\mathcal{E}}(u)^{a_{2} / 2}\|u\|_{L^{p}(\hat{v})}^{1-a_{2}}, \text { where } a_{2}=\left[\frac{1 / p-1 /(q \underline{\delta})}{1 / p+1 /\left(2 \delta_{S}\right)}\right]^{+} .
$$

This proves (3.2) for $q<\infty$. Setting $q \rightarrow \infty$ proves the case when $q=\infty$ as the constant $C$ is independent of $q$.

Suppose in addition that the condition (M.2) is satisfied, we prove that $\left(a_{1}, a_{2}\right)$ is the optimal pair of exponents. We first show that, for any dyadic triangle $S \subseteq \widehat{\mathbb{S}}$, there exists an $h_{S} \in \mathcal{F}(\hat{\mathbb{S}})$ such that

$$
C_{*}^{-1} \leq h_{S} \leq C_{*} \text { on } S, \operatorname{supp}\left(h_{S}\right) \subseteq \tilde{S}, \text { and } \hat{\mathcal{E}}\left(h_{S}\right) \leq C_{*} \hat{v}(S)^{-1 / \delta_{s}},
$$

where $\tilde{S}=\{x \in \hat{\mathbb{S}}: \operatorname{dist}(x, S) \leq \operatorname{diam}(S)\}$.

To see this, suppose first that $S=2^{-1} \mathbb{S}$ for some $m \in \mathbb{Z}$. Let $h$ be the 1 -harmonic function in $\mathbb{S}$ with boundary value

$$
\left.h\right|_{\mathrm{V}_{1}}(x)= \begin{cases}1, & \text { if } x=(0,0), \\ 0, & \text { otherwise }\end{cases}
$$

Let $h(x)=h(-x)$ for $x \in-\mathbb{S}$, and $h(x)=0$ for $x \in \hat{\mathbb{S}} \backslash[\mathbb{S} \cup(-\mathbb{S})]$. Then $h \in \mathcal{F}(\hat{\mathbb{S}})$ and satisfies (3.7). For a general dyadic triangle $S=2^{m} \tau_{i}(\mathbb{S}), i, m \in \mathbb{Z}$, let $h_{S}=$ $h \circ \tau_{i}^{-1} \circ \mathbf{F}_{1}^{m}$. Then $h_{S} \in \mathcal{F}(\hat{\mathbb{S}})$, and the property (3.7) follows from (2.2) and the self-similar property (2.3).

Suppose that (3.4) holds. Let $\left\{S_{m}\right\}_{m \in \mathbb{Z}}$ be the sequence of dyadic triangles in (M.2). For each $m \in \mathbb{Z}$, by the above, there exists an $h_{m} \in \mathcal{F}(\hat{\mathbb{S}})$ such that $h_{m} \sim 1$ on $S_{m}$, $\operatorname{supp}\left(h_{m}\right) \subseteq \tilde{S}_{m}$ and $\hat{\mathcal{E}}\left(h_{m}\right) \lesssim \hat{v}\left(S_{m}\right)^{-1 / \delta_{s}}$, where the notation $A \lesssim B$ means that $A \leq c B$ for some constant $c>0$ independent of $m$, and $A \simeq B$ means that $A \lesssim B$ and $B \lesssim A$. In view of (M.2), it is easily seen that

$$
\left\|h_{m}\right\|_{L^{q}(\hat{\sigma})} \simeq \hat{v}\left(S_{m}\right)^{1 /(q \underline{\delta})},\left\|h_{m}\right\|_{L^{p}(\hat{v})} \simeq \hat{v}\left(S_{m}\right)^{1 / p}, m \rightarrow \infty
$$

and

$$
\left\|h_{m}\right\|_{L^{q}(\hat{\sigma})} \simeq \hat{v}\left(S_{m}\right)^{1 /(q \bar{\delta})},\left\|h_{m}\right\|_{L^{p}(\hat{v})} \simeq \hat{v}\left(S_{m}\right)^{1 / p}, m \rightarrow-\infty .
$$


It follows from the above and (3.4) that

$$
\hat{v}\left(S_{m}\right)^{1 /(q \underline{\delta})} \lesssim \sum_{i=1}^{N} \hat{v}\left(S_{m}\right)^{-b_{i} /\left(2 \delta_{s}\right)+\left(1-b_{i}\right) / p}, \quad m \rightarrow \infty
$$

and

$$
\hat{v}\left(S_{m}\right)^{1 /(q \bar{\delta})} \lesssim \sum_{i=1}^{N} \hat{v}\left(S_{m}\right)^{-b_{i} /\left(2 \delta_{s}\right)+\left(1-b_{i}\right) / p}, \quad m \rightarrow-\infty
$$

These inequalities imply that $\min _{i} b_{i} \leq a_{2} \leq a_{1} \leq \max _{i} b_{i}$.

Remark 3.7 (i) Some comments are desired on the interpretation of the exponents appearing in the inequality (3.2). Recall that, on Euclidean space $\mathbb{R}^{d}$, the celebrated Gagliardo-Nirenberg inequality takes the form

$$
\left\|D^{j} u\right\|_{L^{q}\left(\mathbb{R}^{d}\right)} \leq C\left\|D^{m} u\right\|_{L^{r}\left(\mathbb{R}^{d}\right)}^{a}\|u\|_{L^{p}\left(\mathbb{R}^{d}\right)}^{1-a}
$$

where $a \in[0,1]$ is given by $\frac{1}{q}=\frac{j}{d}+\left(\frac{1}{r}-\frac{m}{d}\right) a+\frac{1-a}{p}$. The case corresponding to setting of Dirichlet forms is the one when $j=0, m=1$ and $r=2$, for which the exponent $a$ is given by

$$
a=\frac{1 / p-1 / q}{1 / p-1 / 2+1 / d} .
$$

Some insights are gained by comparing (3.3) and (3.8):

(a) The exponents $a_{i}, i=1,2$ in (3.2) are determined by the harmonic structure on $\hat{\mathbb{S}}$ (or equivalently the Dirichlet form $\hat{\mathcal{E}}$ ), the configuration parameters $\underline{\delta}$ and $\bar{\delta}$ of the measure $\hat{\sigma}$, and the embedding parameters $p$ and $q$.

(b) The effective Sobolev dimension $d$ of $\hat{\mathbb{S}}$, if exists, should depend only on the harmonic structure. This dependence is expressed in (3.3) as the denominator $1 / p+1 /\left(2 \delta_{s}\right)$. Comparing this to the denominator of $(3.8)$, we see that the Sobolev dimension $d$ should be given by $1 / p-1 / 2+1 / d=1 / p+1 /\left(2 \delta_{s}\right)$, i.e. $d=d_{s}$. This suggests the identification of the spectral dimension $d_{s}$ as the effective Sobolev dimension of $\hat{\mathbb{S}}$. See [31, pp. 44-45] for more comments on $d_{s}$.

(ii) The inequality (3.4) includes the analogue on $\hat{\mathbb{S}}$ of a specific case of the weighted Sobolev inequalities on $\mathbb{R}^{d}$ in [5]. The weighted Sobolev inequalities established in [5] take the form

$$
\left\||x|^{\gamma} u\right\|_{L^{q}\left(\mathbb{R}^{d}\right)} \leq C\left\||x|^{\alpha} D u\right\|_{L^{r}\left(\mathbb{R}^{d}\right)}^{a}\left\||x|^{\beta} u\right\|_{L^{p}\left(\mathbb{R}^{d}\right)}^{1-a}
$$

where $\alpha, \beta, \gamma<0$ satisfy $1 / r+\alpha / d>0,1 / p+\beta / d \geq 1 / q+\gamma / d>0$ and $\frac{1}{q}+\frac{\gamma}{d}=a\left(\frac{1}{r}+\frac{\alpha-1}{d}\right)+(1-a)\left(\frac{1}{p}+\frac{\beta}{d}\right)$. The case corresponding to setting of 
Dirichlet forms is the one when $\alpha=\beta=0, r=2$ and $1 / p \geq 1 / q+\gamma / d_{s}>0$, for which the weighted inequality reads

$$
\|u\|_{L^{q}\left(|x|^{\gamma q} d x\right)} \leq C\|D u\|_{L^{2}(d x)}^{a}\|u\|_{L^{p}(d x)}^{1-a} .
$$

As remarked in Example 3.5.(iii), the analogue on $\hat{\mathbb{S}}$ of $|x|^{\gamma q} d x$ on $\mathbb{R}^{d}$ is a Borel measure $\hat{\sigma}$ on $\hat{\mathbb{S}}$ satisfying the condition (M.1) with $\underline{\delta}, \bar{\delta}$ given by $1 / \underline{\delta}=1 / \bar{\delta}=1+\gamma q / d_{s}$. Therefore, the analogue of (3.9) on $\hat{\mathbb{S}}$ should be $\|u\|_{L^{q}(\hat{\sigma})} \leq C \hat{\mathcal{E}}(u)^{a / 2}\|u\|_{L^{p}(\hat{v})}^{1-a}$ with $a$ given by $\frac{1}{q}+\frac{\gamma}{d_{s}}=a\left(\frac{1}{2}-\frac{1}{d_{s}}\right)+\frac{1-a}{p}$. This coincides with the result of (3.16) since the exponents for the measure $\hat{\sigma}$ are given by $a_{1}=a_{2}=\frac{1 / p-1 / q-\gamma / d_{s}}{1 / p+1 / d_{s}-1 / 2}=a$.

(iii) An additive version of (3.2), which is a corollary of (3.2) and Young's inequality, is derived in [17] for the study of vector fields on resistance spaces.

According to Theorem 3.6, the condition (M.1) is sufficient for the derivation of Sobolev inequalities. The following theorem states that this condition is also necessary for the validity of Sobolev inequalities of the form (3.4) with $q<\infty$.

Theorem 3.8 Let $\hat{\sigma}$ be a Borel measure on $\hat{\mathbb{S}}$. Suppose that there exist some constants $p, q \in(0, \infty), b_{i} \in[0,1]$ where $1 \leq i \leq N$, and $C>0$, such that (3.4) holds for all $u \in \mathcal{F}(\hat{\mathbb{S}})$. Then there exist constants $0<\underline{\delta} \leq \bar{\delta} \leq \infty$ such that the condition (M.1) is satisfied.

Proof Suppose that (3.4) holds. For any dyadic triangle $S \subseteq \hat{\mathbb{S}}$, as shown in the proof of Theorem 3.6, there exists a piecewise harmonic function $h_{S} \in \mathcal{F}(\hat{\mathbb{S}})$ such that

$$
h_{S} \simeq 1 \text { on } S, \operatorname{supp}\left(h_{S}\right) \subseteq \tilde{S} \text {, and } \hat{\mathcal{E}}\left(h_{S}\right) \lesssim \hat{v}(S)^{-1 / \delta_{s}} \text {, }
$$

where the notation $\tilde{S}$ and the relations $\lesssim$ and $\simeq$ are the same as those in the proof of Theorem 3.6. Applying (3.4) to $h_{S}$ gives that

$$
\hat{\sigma}(S)^{1 / q} \lesssim \sum_{i} \hat{v}(S)^{-b_{i} /\left(2 \delta_{s}\right)+\left(1-b_{i}\right) / p}
$$

Since $q<\infty$, it follows from the above that

$$
\sup \{\hat{\sigma}(S): S \text { is a dyadic triangle with } \operatorname{diam}(S)=1\}<\infty .
$$

Therefore, the first part of (M.1) is satisfied with $\bar{\delta}=\infty$.

Furthermore, for any dyadic triangle $S$ with $\operatorname{diam}(S) \geq 1$, by (3.10), $\hat{\sigma}(S)^{1 / q} \lesssim$ $\hat{v}(S)^{1 / p}$ as $\hat{v}(S) \geq 1$. Setting $\underline{\delta}=p / q$ completes the proof.

Applying Theorem 3.6 to the cases when $\hat{\sigma}=\hat{v}$ and when $\hat{\sigma}=\hat{\mu}$, we obtain the following.

Corollary 3.9 Let $1 \leq p \leq q \leq \infty, q \geq 2$. Then 
(a) The inequality (3.2) holds with $\hat{\sigma}=\hat{v}$ and $a_{1}=a_{2}=\frac{1 / p-1 / q}{1 / p+1 /\left(2 \delta_{s}\right)} \in[0,1)$. In particular,

$$
\max _{\hat{\mathbb{S}}} u \leq C \hat{\mathcal{E}}(u)^{a / 2}\|u\|_{L^{p}(\hat{v})}^{1-a}, \quad u \in \mathcal{F}(\hat{\mathbb{S}})
$$

with $a=\frac{1 / p}{1 / p+1 /\left(2 \delta_{s}\right)}$. Conversely, the inequality (3.2) holds for all $u \in \mathcal{F}(\hat{\mathbb{S}})$ if and only if $a_{1}=a_{2}=\frac{1 / p-1 / q}{1 / p+1 /\left(2 \delta_{s}\right)}$.

(b) The inequality (3.2) holds with $\hat{\sigma}=\hat{\mu}$. The pair $\left(a_{1}, a_{2}\right)$ given by (3.3) is optimal, where $\underline{\delta}=1$ and $\bar{\delta}=\delta_{s}$.

Proof The only thing needs a proof is that $\bar{\delta}=\delta_{s}$ in (b). Clearly,

$$
1 / \bar{\delta}=\inf _{\omega \in \mathrm{W}_{*}} \liminf _{m \rightarrow \infty}\left[-\frac{1}{m \log 3} \log \mu\left(\mathbb{S}_{[\omega]_{m}}\right)\right] .
$$

We show that

$$
\sup _{\omega \in \mathrm{W}_{*}} \lim _{m \rightarrow \infty}\left[\operatorname{tr}\left(\mathbf{A}_{[\omega]_{m}}^{\mathrm{t}} \mathbf{P} \mathbf{A}_{[\omega]_{m}}\right)\right]^{1 / m}=(3 / 5)^{2},
$$

from which the conclusion follows immediately.

Let $\mathbf{Y}_{i}=\mathbf{P}^{\mathrm{t}} \mathbf{A}_{i} \mathbf{P}, i=1,2,3$. Then $\mathbf{Y}_{i}, i=1,2,3$ have the same eigenvalues $\{0,1 / 5,3 / 5\}$. It is easily seen that $\mathbf{A}_{[\omega]_{m}}^{\mathrm{t}} \mathbf{P A}_{[\omega]_{m}}=\mathbf{Y}_{[\omega]_{m}}^{\mathrm{t}} \mathbf{Y}_{[\omega]_{m}}$ for every $m \in \mathbb{N}_{+}$ and every $\omega \in \mathrm{W}_{m}$, where $\mathbf{Y}_{[\omega]_{m}}=\mathbf{Y}_{\omega_{m}} \cdots \mathbf{Y}_{\omega_{2}} \mathbf{Y}_{\omega_{1}}$. Therefore, $\operatorname{tr}\left(\mathbf{Y}_{[\omega]_{m}}^{\mathrm{t}} \mathbf{Y}_{[\omega]_{m}}\right) \leq$ $C_{*}(3 / 5)^{2 m}$, which implies that

$$
\sup _{\omega \in \mathrm{W}_{*}} \lim _{m \rightarrow \infty}\left[\operatorname{tr}\left(\mathbf{Y}_{[\omega]_{m}}^{\mathrm{t}} \mathbf{Y}_{[\omega]_{m}}\right)\right]^{1 / m} \leq(3 / 5)^{2}
$$

For the reverse, let $\omega=111 \ldots \in \mathrm{W}_{*}$. Then $\lim _{m \rightarrow \infty}\left[\operatorname{tr}\left(\mathbf{Y}_{[\omega]_{m}}^{\mathrm{t}} \mathbf{Y}_{[\omega]_{m}}\right)\right]^{1 / m}=(3 / 5)^{2}$. This proves (3.12).

Remark 3.10 Setting $p=1, q=2$ in (3.11) gives the Nash inequality on $\hat{\mathbb{S}}$ (see [8, Theorem 4.1])

$$
\|u\|_{L^{2}(\hat{v})}^{2+4 / d_{s}} \leq C \hat{\mathcal{E}}(u)\|u\|_{L^{1}(\hat{v})}^{4 / d_{s}}, \quad u \in \mathcal{F}(\hat{\mathbb{S}})
$$

Conclusions similar to that of Theorem 3.6 hold when the roles of $\hat{\sigma}$ and $\hat{v}$ are exchanged. More specifically, let $\hat{\sigma}$ be a Borel measure on $\hat{\mathbb{S}}$ satisfying the following condition: there exist constants $C_{\hat{\sigma}} \geq 1$ and $0<\underline{\delta} \leq \bar{\delta}<\infty$ such that

$$
\left\{\begin{array}{l}
C_{\hat{\sigma}}^{-1} \hat{v}(S)^{1 / \underline{\delta}} \leq \hat{\sigma}(S), \quad \text { if } 0<\operatorname{diam}(S)<1 \\
C_{\hat{\sigma}}^{-1} \hat{v}(S)^{1 / \bar{\delta}} \leq \hat{\sigma}(S), \quad \text { if } \operatorname{diam}(S) \geq 1
\end{array}\right.
$$

for any dyadic triangle $S \subseteq \hat{\mathbb{S}}$. For measures $\hat{\sigma}$ satisfying (M.1'), we have Theorems 3.11 and 3.13 below, of which the proofs will be omitted as they are are similar to those of Theorems 3.6 and 3.8. 
Theorem 3.11 Let $1 \leq p \leq q \leq \infty, q \geq 2$. Suppose that $\hat{\sigma}$ is a Borel measure on $\hat{\mathbb{S}}$ satisfying the condition (M.1'). Then

$$
\|u\|_{L^{q}(\hat{v})} \leq C \sum_{i=1,2} \hat{\mathcal{E}}(u)^{a_{i} / 2}\|u\|_{L^{p}(\hat{\sigma})}^{1-a_{i}}, \quad u \in \mathcal{F}(\hat{\mathbb{S}}),
$$

where

$$
a_{1}=\left[\frac{1 /(p \underline{\delta})-1 / q}{1 /(p \underline{\delta})+1 /\left(2 \delta_{s}\right)}\right]^{+}, a_{2}=\left[\frac{1 /(p \bar{\delta})-1 / q}{1 /(p \bar{\delta})+1 /\left(2 \delta_{s}\right)}\right]^{+},
$$

and $C>0$ is a constant depending only on the constant $C_{\hat{\sigma}}$ in (M.1').

Moreover, if there exists a sequence $\left\{S_{m}\right\}_{m \in \mathbb{Z}}$ of dyadic triangles such that

$$
\lim _{m \rightarrow-\infty} \operatorname{diam}\left(S_{m}\right)=0, \lim _{m \rightarrow \infty} \operatorname{diam}\left(S_{m}\right)=\infty
$$

and

$$
1 / \underline{\delta}=\lim _{m \rightarrow-\infty} \frac{\log \hat{\sigma}\left(S_{m}\right)}{\log \hat{v}\left(S_{m}\right)}, 1 / \bar{\delta}=\lim _{m \rightarrow \infty} \frac{\log \hat{\sigma}\left(S_{m}\right)}{\log \hat{v}\left(S_{m}\right)}
$$

then the pair of exponents given by (3.14) is optimal in the following sense: if

$$
\|u\|_{L^{q}(\hat{v})} \leq C \sum_{i=1}^{N} \hat{\mathcal{E}}(u)^{b_{i} / 2}\|u\|_{L^{p}(\hat{\sigma})}^{1-b_{i}}, \quad u \in \mathcal{F}(\hat{\mathbb{S}})
$$

for some constants $b_{i} \in[0,1]$ where $1 \leq i \leq N, N \in \mathbb{N}_{+}$and $C>0$ independent of $u$, then $\min _{i} b_{i} \leq a_{2} \leq a_{1} \leq \max _{i} b_{i}$.

Remark 3.12 Theorems 3.6 and 3.11 can be easily combined to yield the following

$$
\|u\|_{L^{q}\left(\hat{\sigma}_{2}\right)} \leq C \sum_{i=1,2} \hat{\mathcal{E}}(u)^{a_{i} / 2}\|u\|_{L^{p}\left(\hat{\sigma}_{1}\right)}^{1-a_{i}}, \quad u \in \mathcal{F}(\hat{\mathbb{S}})
$$

where $\hat{\sigma}_{2}$ satisfies (M.1) with $\bar{\delta}=\bar{\delta}_{2}, \underline{\delta}=\underline{\delta}_{2}, \hat{\sigma}_{1}$ satisfies (M.1') with $\bar{\delta}=\bar{\delta}_{1}, \underline{\delta}=\underline{\delta}_{1}$, and

$$
a_{1}=\left[\frac{1 /\left(p \underline{\delta}_{1}\right)-1 /\left(q \bar{\delta}_{2}\right)}{1 /\left(p \underline{\delta}_{1}\right)+1 /\left(2 \delta_{2}\right)}\right]^{+}, a_{2}=\left[\frac{1 /\left(p \bar{\delta}_{1}\right)-1 /\left(q \underline{\delta}_{2}\right)}{1 /\left(p \bar{\delta}_{1}\right)+1 /\left(2 \delta_{s}\right)}\right]^{+} \text {. }
$$

Theorem 3.13 Let $\hat{\sigma}$ be a Borel measure on $\hat{\mathbb{S}}$. Suppose that there exist some constants $p, q \in(0, \infty), b_{i} \in[0,1]$ where $1 \leq i \leq N$ and $C>0$ such that (3.15) holds for all $u \in \mathcal{F}(\hat{\mathbb{S}})$. Then there exist constants $0<\underline{\delta} \leq \bar{\delta} \leq \infty$ such that the condition (M.1') is satisfied.

Corollary 3.14 The inequality (3.13) holds with $\hat{\sigma}=\hat{\mu}$. The pair $\left(a_{1}, a_{2}\right)$ of exponents given by (3.14) is optimal, where the constants $\bar{\delta}=1$ and $\underline{\delta}$ is given by $1 / \underline{\delta}=1 / \delta_{s}+2$. 
Remark 3.15 The value of $\underline{\delta}$ in Corollary 3.14 follows from the fact that

$$
\inf _{\omega \in \mathrm{W}_{*}} \lim _{m \rightarrow \infty}\left[\operatorname{tr}\left(\mathbf{A}_{[\omega]_{m}}^{\mathrm{t}} \mathbf{P} \mathbf{A}_{[\omega]_{m}}\right)\right]^{1 / m}=3 / 25
$$

which will be given in another work by the present authors.

We end this section with the corresponding Sobolev inequalities on the compact gasket $\mathbb{S}$, whose proof shall be omitted. Let $\sigma$ be a finite Borel measure on $\mathbb{S}$. For the compact gasket, only the first part of the condition (M.1) is relevant, i.e.

$$
\sigma\left(\mathbb{S}_{[\omega]_{m}}\right) \leq C_{\sigma} v\left(\mathbb{S}_{[\omega]_{m}}\right)^{1 / \bar{\delta}}, \text { for all } \omega \in \mathrm{W}_{*} \text { and all } m \in \mathbb{N}
$$

where $C_{\sigma}>0$ and $\bar{\delta} \in[1, \infty]$ are constants depending only on the Borel measure $\sigma$. Similarly, we only need the first part of the condition (M.1'), i.e.

$$
C_{\sigma}^{-1} v\left(\mathbb{S}_{[\omega]_{m}}\right)^{1 / \underline{\delta}} \leq \sigma\left(\mathbb{S}_{[\omega]_{m}}\right), \text { for all } \omega \in \mathrm{W}_{*} \text { and all } m \in \mathbb{N}
$$

where $\underline{\delta} \in(0, \infty]$ is a constant depending only on the Borel measure $\sigma$.

Theorem 3.16 Let $1 \leq p \leq q \leq \infty, q \geq 2$, and let $\sigma$ be a finite Borel measure on $\mathbb{S}$. (a) Suppose that $\sigma$ satisfies (3.17). Then for any $u \in \mathcal{F}(\mathbb{S})$,

$$
\|u-c\|_{L^{q}(\sigma)} \leq C \mathcal{E}(u)^{a / 2}\|u-c\|_{L^{p}(\nu)}^{1-a} .
$$

where $c$ is any constant satisfying $\min _{\mathbb{S}} u \leq c \leq \max _{\mathbb{S}} u$, and

$$
a=\left[\frac{1 / p-1 /(q \bar{\delta})}{1 / p+1 /\left(2 \delta_{s}\right)}\right]^{+},
$$

and $C>0$ is a constant depending only on the constant $C_{\sigma}$ in (3.17). Therefore, for any $u \in \mathcal{F}(\mathbb{S})$,

$$
\|u\|_{L^{q}(\sigma)} \leq C\left[\mathcal{E}(u)^{a / 2}\|u\|_{L^{p}(v)}^{1-a}+\|u\|_{L^{p}(v)}\right]
$$

Moreover, the exponent a given by (3.20) is optimal in the sense that if (3.19) holds for some $a \in[0,1]$, then $a \geq\left[\frac{1 / p-1 /(q \bar{\delta})}{1 / p+1 /\left(2 \delta_{s}\right)}\right]^{+}$.

(b) Suppose that $\sigma$ satisfies (3.18). Then the conclusions of (a) hold when $\sigma$ and $v$ are exchanged and the exponent (3.20) is replaced by

$$
a=\left[\frac{1 /(p \underline{\delta})-1 / q}{1 /(p \underline{\delta})+1 /\left(2 \delta_{s}\right)}\right]^{+},
$$


Remark 3.17 Setting $\sigma=v, \underline{\delta}=\bar{\delta}=1$ and $p=1, q=2$ in (3.21) gives the Nash inequality on $\mathbb{S}$ (see [8, Theorem 4.4] or [24, Theorem 5.3.3])

$$
\begin{aligned}
\|u\|_{L^{2}(v)}^{2+4 / d_{s}} & \leq C\left[\mathcal{E}(u)+\|u\|_{L^{1}(v)}^{2}\right]\|u\|_{L^{1}(v)}^{4 / d_{s}} \\
& \leq C\left[\mathcal{E}(u)+\|u\|_{L^{2}(v)}^{2}\right]\|u\|_{L^{1}(v)}^{4 / d_{s}}, \quad u \in \mathcal{F}(\mathbb{S}) .
\end{aligned}
$$

Corollary 3.18 For any $u \in \mathcal{F}(\mathbb{S})$,

$$
\|u\|_{L^{2}(\mu)} \leq C\left[\mathcal{E}(u)^{\left(d_{s}-1\right) / 2}\|u\|_{L^{2}(v)}^{2-d_{s}}+\|u\|_{L^{2}(v)}\right] .
$$

If $u \in \mathcal{F}\left(\mathbb{S} \backslash \mathrm{V}_{0}\right)$ in addition, then by (3.19) with $c=0$,

$$
\|u\|_{L^{2}(\mu)} \leq C \mathcal{E}(u)^{\left(d_{s}-1\right) / 2}\|u\|_{L^{2}(v)}^{2-d_{s}} .
$$

\section{Semi-linear parabolic PDEs}

In this section, we study a type of semi-linear parabolic equations on $\mathbb{S}$, for which energy estimates and existence and uniqueness of solutions are established (Theorem 4.16). Moreover, the regularity of solutions to these PDEs is derived under additional conditions.

We consider the following initial-boundary value problem for semi-linear parabolic PDEs (see Definition 4.13 below for a precise interpretation)

$$
\left\{\begin{array}{c}
\partial_{t} u d v=\mathcal{L} u d v+f(t, x, u, \nabla u) d \mu, \quad \text { in }(0, T] \times\left(\mathbb{S} \backslash \mathrm{V}_{0}\right), \\
u=0 \text { on }(0, T] \times \mathrm{V}_{0}, \quad u(0)=\psi,
\end{array}\right.
$$

where $\psi \in L^{2}(v)$, and the coefficient $f:[0, T] \times \mathbb{S} \times \mathbb{R}^{2} \rightarrow \mathbb{R}$ satisfies the following:

(i) There exists a constant $K>0$ such that

$$
|f(t, x, y, z)-f(t, x, \bar{y}, \bar{z})| \leq K(|y-\bar{y}|+|z-\bar{z}|)
$$

for all $(t, x) \in[0, T] \times \mathbb{S},(y, z),(\bar{y}, \bar{z}) \in \mathbb{R}^{2}$

(ii) $f(\cdot, 0,0) \in L^{2}\left(0, T ; L^{2}(\mu)\right)$, that is,

$$
\|f(\cdot, 0,0)\|_{L^{2}\left(0, T ; L^{2}(\mu)\right)}^{2}=\int_{0}^{T} \int_{\mathbb{S}} f(t, x, 0,0)^{2} \mu(d x) d t<\infty .
$$

Remark 4.1 There exist different formulations of non-linear PDEs on fractals. For example, a type of non-linear equations on fractals was considered by in [19], where the non-linearity $f(\nabla u)$ is a bounded mapping $f: L^{2}(\mu) \rightarrow L^{2}(v)$. The equations studied there are essentially defined via a single measure (the Hausdorff measure $v$ ). Therefore, the PDEs studied in this paper are different in essence from those considered in [19] in the way the gradients interact with the equations. 
From now on, we shall use the notation $\langle f, g\rangle_{\lambda}=\int_{\mathbb{S}} f g d \lambda$ for any Borel measure $\lambda$ on $\mathbb{S}$ and any $\lambda$-a.e. defined functions $f, g$ on $\mathbb{S}$, whenever the integral is well-defined. As in the previous section, we denote by $C_{*}$ a generic universal constant which may vary on different occasions.

Let $\left\{P_{t}\right\}_{t \geq 0}$ be the Markov semigroup associated with the killed Brownian motion on $\mathbb{S}$, the diffusion processes associated with the Dirichlet form $\left(\mathcal{E}, \mathcal{F}\left(\mathbb{S} \backslash \mathrm{V}_{0}\right)\right) .\left\{P_{t}\right\}_{t \geq 0}$ admits a jointly continuous heat kernel $p(t, x, y)$, which is $C^{\infty}$ in $t$ (cf. [3, Theorem 1.5]). The following result on heat kernel and resolvent kernel estimate was first proved in [3, Theorems 1.5, 1.8].

Lemma 4.2 For each $t>0$

$$
p(t, x, y) \leq C_{*} t^{-d_{s} / 2}, \quad x, y \in \mathbb{S},
$$

is valid. Let $\rho_{\alpha}, \alpha>0$ be the $\alpha$-resolvent kernel of $\mathcal{L}$, that is,

$$
\rho_{\alpha}(x, y)=\int_{0}^{\infty} e^{-\alpha t} p(t, x, y) d t, \quad x, y \in \mathbb{S} .
$$

Then $\rho_{\alpha}(\cdot, \cdot)$ is Lipschitz continuous with respect to the resistance metric, i.e.

$$
\left|\rho_{\alpha}(x, z)-\rho_{\alpha}(y, z)\right| \leq C_{\alpha} R(x, y), \quad x, y, z \in \mathbb{S},
$$

for some constant $C_{\alpha}>0$ depending only on $\alpha$.

In view of the joint continuity of $p(t, x, y)$, the definition below is legitimate.

Definition 4.3 For any Radon measure $\lambda$ on $\mathbb{S}$, we define $P_{t} \lambda(x)=\int_{\mathbb{S}} p(t, x, y) \lambda(d y)$, $x \in \mathbb{S}, t \in(0, \infty)$.

Remark 4.4 (i) Let $\lambda$ be a Radon measure on $\mathbb{S}$. By the symmetry of $p(t, \cdot, \cdot)$, it is easy to see that $\left\langle P_{t}(g \lambda), f\right\rangle_{\nu}=\left\langle g, P_{t} f\right\rangle_{\lambda}$ for all $f \in L^{2}(\nu), g \in L^{1}(\lambda)$.

(ii) For any Radon measure $\lambda$ on $\mathbb{S}$, we have $P_{t} \lambda \in \operatorname{Dom}(\mathcal{L})$ for $t>0$. In fact, since $p(t, x, y) \in C((0, \infty) \times \mathbb{S} \times \mathbb{S})$, we have $P_{t / 2} \lambda \in C(\mathbb{S})$, which implies that $P_{t} \lambda=P_{t / 2}\left(P_{t / 2} \lambda\right) \in \operatorname{Dom}(\mathcal{L})$. Moreover, $P_{t} \lambda \in C^{1}\left(0, \infty ; L^{2}(v)\right)$ and $\frac{d}{d t} P_{t} \lambda=\mathcal{L} P_{t} \lambda$.

(iii) Notice that, due to the singularity between $v$ and $\mu$, the contractivity $\left\|P_{t}(g \mu)\right\|_{L^{2}(v)}$ $\leq\|g\|_{L^{2}(\mu)}, t>0$ is no longer valid in general. In fact, for $g \in L^{2}(\mu), g \neq 0$, we have

$$
\lim _{t \rightarrow 0}\left\|P_{t}(g \mu)\right\|_{L^{2}(v)}=\infty .
$$

To see this, suppose contrarily that $\lim _{t \rightarrow 0}\left\|P_{t}(g \mu)\right\|_{L^{2}(v)}=\sup _{t>0}\left\|P_{t}(g \mu)\right\|_{L^{2}(v)}$ $<\infty$. Then there exists a unique $g_{0} \in L^{2}(\nu)$ such that $\lim _{t \rightarrow 0} P_{t}(g \mu)=g_{0}$ weakly in $L^{2}(v)$. On the other hand, for any $v \in \mathcal{F}\left(\mathbb{S} \backslash \mathrm{V}_{0}\right)$, we have

$$
\left\langle g_{0}, v\right\rangle_{v}=\lim _{t \rightarrow 0}\left\langle P_{t}(g \mu), v\right\rangle_{v}=\lim _{t \rightarrow 0}\left\langle g, P_{t} v\right\rangle_{\mu}=\langle g, v\rangle_{\mu}
$$


where the last equality follows from the uniform convergence $\lim _{t \rightarrow 0} P_{t} v=v$ as a consequence of the convergence in $\mathcal{F}\left(\mathbb{S} \backslash \mathrm{V}_{0}\right)$. By the density of $\mathcal{F}\left(\mathbb{S} \backslash \mathrm{V}_{0}\right)$ in $C(\mathbb{S})$, it is seen that $g \mu=g_{0} \nu$, which contradicts the fact that $\nu$ and $\mu$ are mutually singular.

To study the semi-linear parabolic PDEs (4.1), let us first investigate the formal integral

$$
\int_{0}^{t} P_{t-s}(g(s) \mu) d s
$$

which is the formal solution to the equation $\partial_{t} u d v=\mathcal{L} u d v+g(t) d \mu$. Since $P_{t}$ is not bounded from $L^{2}(\mu)$ to $L^{2}(v)$ (cf. Remark 4.4.(iii) above), there is a singularity in the integrand of (4.2) at $s=t$. We shall show that (4.2) is a well-defined function in the space $L^{\infty}\left(0, T ; L^{2}(v)\right) \cap L^{2}\left(0, T ; \mathcal{F}\left(\mathbb{S} \backslash \mathrm{V}_{0}\right)\right)$, and is jointly Hölder continuous if $g(t)$ is uniformly bounded in $L^{2}(\mu)$. To formulate the results, it is convenient to introduce several definitions.

Definition 4.5 For any $v \in L^{2}(v)$, define

$$
\|v\|_{\mathcal{F}^{-1}}=\sup \left\{\langle u, v\rangle_{v}: u \in \mathcal{F}(\mathbb{S}),\|u\|_{\mathcal{F}} \leq 1\right\}
$$

where

$$
\|u\|_{\mathcal{F}}=\left[\|u\|_{L^{2}(v)}^{2}+\mathcal{E}(u)\right]^{1 / 2}
$$

The space $\mathcal{F}^{-1}(\mathbb{S})$ is defined to be the $\|\cdot\|_{\mathcal{F}^{-1}}$-completion of $L^{2}(v)$.

Definition 4.6 Let $u \in L^{2}(0, T ; \mathcal{F}(\mathbb{S}))$; that is, $\int_{0}^{T} \mathcal{E}_{1}(u(t)) d t<\infty$. An $(\mathcal{F}(\mathbb{S})$ valued) function $u$ is said to have a weak derivative $\partial_{t} u$ in $L^{2}\left(0, T ; \mathcal{F}^{-1}(\mathbb{S})\right)$, if $\partial_{t} u$ is an $\mathcal{F}^{-1}(\mathbb{S})$-valued function on $[0, T]$ satisfying

$$
\left(\int_{0}^{T}\left\|\partial_{t} u(t)\right\|_{\mathcal{F}^{-1}}^{2} d t\right)^{1 / 2}<\infty \text { and } \int_{0}^{T}\left\langle u(t), \partial_{t} v(t)\right\rangle_{\nu} d t=-\int_{0}^{T}\left\langle\partial_{t} u(t), v(t)\right\rangle_{\nu} d t
$$

for all $v \in C^{1}(0, T ; \mathcal{F}(\mathbb{S}))$ with $v(0)=v(T)=0$.

The following lemma can be easily shown by a mollifier argument similar to that of [7, Theorem 3, Section 5.9].

Lemma 4.7 Suppose that $u \in L^{2}\left(0, T ; \mathcal{F}\left(\mathbb{S} \backslash \mathrm{V}_{0}\right)\right)$ has a weak derivative $\partial_{t} u \in$ $L^{2}\left(0, T ; \mathcal{F}^{-1}(\mathbb{S})\right)$. Then

(a) $u \in C\left(0, T ; L^{2}(v)\right)$; (b) The function $t \mapsto\|u(t)\|_{L^{2}(v)}^{2}$ is absolutely continuous, and $\frac{d}{d t}\|u(t)\|_{L^{2}(v)}^{2}=2\left\langle\partial_{t} u(t), u(t)\right\rangle_{v}$ for a.e. $t \in[0, T]$.

We derive properties of the convolution (4.2) in the following lemmas. 
Lemma 4.8 Let $g \in L^{2}\left(0, T ; L^{2}(\mu)\right)$. For each $\delta \in(0, T)$, let

$$
u_{\delta}(t)=\int_{0}^{(t-\delta)^{+}} P_{t-s}(g(s) \mu) d s, \quad t \in[0, T]
$$

Then $u_{\delta} \in L^{\infty}\left(0, T ; L^{2}(v)\right) \cap L^{2}\left(0, T ; \mathcal{F}\left(\mathbb{S} \backslash V_{0}\right)\right)$ and

$$
\left\|u_{\delta}\right\|_{L^{\infty}\left(0, T ; L^{2}(v)\right)}^{2}+\int_{0}^{T} e^{C_{\epsilon}(T-s)} \mathcal{E}\left(u_{\delta}(s)\right) d s \leq \epsilon \int_{0}^{T} e^{C_{\epsilon}(T-s)}\|g(s)\|_{L^{2}(\mu)}^{2} d s,
$$

for any $\epsilon>0$, where $C_{\epsilon}>0$ is a constant depending only on $\epsilon$. Moreover,

$$
\left\|\partial_{t} u_{\delta}\right\|_{L^{2}\left(0, T ; \mathcal{F}^{-1}\right)} \leq C_{*}\|g\|_{L^{2}\left(0, T ; L^{2}(\mu)\right)} .
$$

Proof It is convenient to set $g(t)=0$ for $t<0$. Clearly, $u_{\delta}(t) \in \operatorname{Dom}(\mathcal{L}), t \in[0, T]$. For each $s \in(0, T)$, since $t \mapsto P_{t-s}(g(s) \mu)=P_{t-s-\delta}\left[P_{\delta}(g(s) \mu)\right], t \in(s+\delta, T)$ is a continuously differentiable $L^{2}(v)$-valued function, we see that $u_{\delta} \in C^{1}\left(\delta, T ; L^{2}(v)\right)$ and

$$
\partial_{t} u_{\delta}(t)=P_{\delta}(g(t-\delta) \mu)+\int_{0}^{t-\delta} \mathcal{L} P_{t-s}(g(s) \mu) d s=\mathcal{L} u_{\delta}(t)+P_{\delta}(g(t-\delta) \mu) .
$$

For any $\epsilon>0$ and each $t \in(0, T)$, testing (4.4) against $u_{\delta}$ and applying Corollary 3.18 gives that

$$
\begin{aligned}
\frac{1}{2} \frac{d}{d t}\left\|u_{\delta}(t)\right\|_{L^{2}(v)}^{2}= & \left\langle P_{\delta}(g(t-\delta) \mu), u_{\delta}(t)\right\rangle_{v}-\mathcal{E}\left(u_{\delta}(t)\right) \\
= & \left\langle g(t-\delta), P_{\delta}\left(u_{\delta}(t)\right)\right\rangle_{\mu}-\mathcal{E}\left(u_{\delta}(t)\right) \\
\leq & C_{\epsilon} \mathcal{E}\left[P_{\delta}\left(u_{\delta}(t)\right)\right]^{d_{s}-1}\left\|P_{\delta}\left(u_{\delta}(t)\right)\right\|_{L^{2}(v)}^{2\left(2-d_{s}\right)} \\
& -\mathcal{E}\left(u_{\delta}(t)\right)+\epsilon\|g(t-\delta)\|_{L^{2}(\mu)}^{2} \\
\leq & C_{\epsilon} \mathcal{E}\left(u_{\delta}(t)\right)^{d_{s}-1}\left\|u_{\delta}(t)\right\|_{L^{2}(v)}^{2\left(2-d_{s}\right)}-\mathcal{E}\left(u_{\delta}(t)\right)+\epsilon\|g(t-\delta)\|_{L^{2}(\mu)}^{2} \\
\leq & C_{\epsilon}\left\|u_{\delta}(t)\right\|_{L^{2}(v)}^{2}-\frac{1}{2} \mathcal{E}\left(u_{\delta}(t)\right)+\epsilon\|g(t-\delta)\|_{L^{2}(\mu)}^{2},
\end{aligned}
$$

where $C_{\epsilon}>0$ denotes a generic constant depending only on $\epsilon$ which may vary on different occasions. By Grönwall's inequality and the fact that $u_{\delta}(t)=0, t \in[0, \delta]$, we deduce

$$
\left\|u_{\delta}(t)\right\|_{L^{2}(v)}^{2}+\int_{0}^{t} e^{C_{\epsilon}(t-s)} \mathcal{E}\left(u_{\delta}(s)\right) d s \leq \epsilon \int_{0}^{(t-\delta)^{+}} e^{C_{\epsilon}(t-s)}\|g(s)\|_{L^{2}(\mu)}^{2} d s .
$$

By (4.4) again, for any $v \in \mathcal{F}\left(\mathbb{S} \backslash \mathrm{V}_{0}\right)$,

$$
\left|\left\langle\partial_{t} u_{\delta}(t), v\right\rangle_{\nu}\right| \leq\left|\left\langle g(t-\delta), P_{\delta} v\right\rangle_{\mu}\right|+\mathcal{E}\left(u_{\delta}(t)\right)^{1 / 2} \mathcal{E}(v)^{1 / 2}
$$




$$
\begin{aligned}
& \leq C_{*}\|g(t-\delta)\|_{L^{2}(\mu)} \mathcal{E}\left(P_{\delta} v\right)^{1 / 2}+\mathcal{E}\left(u_{\delta}(t)\right)^{1 / 2} \mathcal{E}(v)^{1 / 2} \\
& \leq C_{*}\left[\|g(t-\delta)\|_{L^{2}(\mu)}+\mathcal{E}\left(u_{\delta}(t)\right)^{1 / 2}\right]\|v\|_{\mathcal{F}} .
\end{aligned}
$$

The above inequality also holds for $v \in \mathcal{F}(\mathbb{S})$. This can be seen by considering the $\mathcal{F}$-orthogonal projection of $v$ on $\mathcal{F}\left(\mathbb{S} \backslash \mathrm{V}_{0}\right)$. Therefore,

$$
\left\|\partial_{t} u_{\delta}(t)\right\|_{\mathcal{F}^{-1}} \leq C_{*}\left[\|g(t-\delta)\|_{L^{2}(\mu)}+\mathcal{E}\left(u_{\delta}(t)\right)^{1 / 2}\right], \quad t \in(\delta, T],
$$

which, together with (4.5), implies the desired estimate for $\left\|\partial_{t} u_{\delta}\right\|_{L^{2}\left(0, T ; \mathcal{F}^{-1}\right)}$.

\section{Lemma 4.9 The limit}

$$
u(t)=\lim _{\delta \rightarrow 0} \int_{0}^{(t-\delta)^{+}} P_{t-s}(g(s) \mu) d s,
$$

exists with respect to the norm $\|\cdot\|_{L^{\infty}\left(0, T ; L^{2}(v)\right)}+\|\cdot\|_{L^{2}(0, T ; \mathcal{F})}$, and satisfies

$$
\|u\|_{L^{\infty}\left(0, T ; L^{2}(v)\right)}^{2}+\int_{0}^{T} e^{C_{\epsilon}(T-s)} \mathcal{E}(u(s)) d s \leq \epsilon \int_{0}^{T} e^{C_{\epsilon}(T-s)}\|g(s)\|_{L^{2}(\mu)}^{2} d s .
$$

Moreover, $u(t)$ has a weak derivative $\partial_{t} u$ in $L^{2}\left(0, T ; \mathcal{F}^{-1}\right)$, and

$$
\left\|\partial_{t} u\right\|_{L^{2}\left(0, T ; \mathcal{F}^{-1}\right)} \leq C_{*}\|g\|_{L^{2}\left(0, T ; L^{2}(\mu)\right)} \cdot
$$

Proof As before, we set $g(t)=0$ for $t<0$. Let $\delta, \delta^{\prime} \in(0, T)$ and $w=u_{\delta}-u_{\delta^{\prime}}$, where $u_{\delta}$ are the functions defined by (4.3). By (4.4), we have

$$
\partial_{t} w=\mathcal{L} w+P_{\delta}\left[\left(g(t-\delta)-g\left(t-\delta^{\prime}\right)\right) \mu\right]+\left(P_{\delta}-P_{\delta^{\prime}}\right)\left(g\left(t-\delta^{\prime}\right) \mu\right),
$$

from which it follows that

$$
\begin{aligned}
\frac{1}{2} \frac{d}{d t}\|w(t)\|_{L^{2}(v)}^{2}= & -\mathcal{E}(w(t))+\left\langle P_{\delta}\left[\left(g(t-\delta)-g\left(t-\delta^{\prime}\right)\right) \mu\right], w(t)\right\rangle_{\nu} \\
& +\left\langle\left(P_{\delta}-P_{\delta^{\prime}}\right)\left(g\left(t-\delta^{\prime}\right) \mu\right), w(t)\right\rangle_{\nu} .
\end{aligned}
$$

The first term on the right hand side of (4.7) can be estimated in the same way as in the proof of Lemma 4.8, which yields that

$$
\begin{aligned}
\left\langle P_{\delta}\left[\left(g(t-\delta)-g\left(t-\delta^{\prime}\right)\right) \mu\right], w(t)\right\rangle_{\nu} \leq & \frac{1}{2}\left\|g(t-\delta)-g\left(t-\delta^{\prime}\right)\right\|_{L^{2}(\mu)}^{2} \\
& +\frac{1}{2} \mathcal{E}(w(t))^{d_{s}-1}\|w(t)\|_{L^{2}(v)}^{2\left(2-d_{s}\right)} .
\end{aligned}
$$

For the second term on the right hand side of (4.7), we have

$$
\left\langle\left(P_{\delta}-P_{\delta^{\prime}}\right)\left(g\left(t-\delta^{\prime}\right) \mu\right), w(t)\right\rangle_{\nu}
$$




$$
\leq C_{*} \mathcal{E}\left(\left(P_{\delta}-P_{\delta^{\prime}}\right) w(t)\right)^{\left(d_{s}-1\right) / 2}\left\|\left(P_{\delta}-P_{\delta^{\prime}}\right) w(t)\right\|_{L^{2}(v)}^{2-d_{s}}\left\|g\left(t-\delta^{\prime}\right)\right\|_{L^{2}(\mu)} .
$$

By the spectral decomposition,

$$
\begin{aligned}
\left\|\left(P_{\delta}-P_{\delta^{\prime}}\right) w(t)\right\|_{L^{2}(v)}^{2} & =\int_{0}^{\infty}\left(e^{-\lambda \delta}-e^{-\lambda \delta^{\prime}}\right)^{2} d\left\|E_{\lambda} w(t)\right\|_{L^{2}(v)}^{2} \\
& \leq \int_{0}^{\infty}\left(1-e^{-\lambda\left|\delta-\delta^{\prime}\right|}\right)^{2} d\left\|E_{\lambda} w(t)\right\|_{L^{2}(v)}^{2} \\
& \leq\left|\delta-\delta^{\prime}\right| \int_{0}^{\infty} \lambda d\left\|E_{\lambda} w(t)\right\|_{L^{2}(v)}^{2} \\
& =\left|\delta-\delta^{\prime}\right| \mathcal{E}(w(t)),
\end{aligned}
$$

which, together with the fact that $\mathcal{E}\left(\left(P_{\delta}-P_{\delta^{\prime}}\right) w(t)\right) \leq \mathcal{E}(w(t))$, implies that

$$
\left\langle\left(P_{\delta}-P_{\delta^{\prime}}\right)\left(g\left(t-\delta^{\prime}\right) \mu\right), w(t)\right\rangle_{\nu} \leq C_{*}\left|\delta-\delta^{\prime}\right|^{1-d_{s} / 2} \mathcal{E}(w(t))^{1 / 2}\left\|g\left(t-\delta^{\prime}\right)\right\|_{L^{2}(\mu)} .
$$

Therefore, we deduce from (4.7) that

$$
\begin{aligned}
\frac{d}{d t}\|w(t)\|_{L^{2}(v)}^{2} \leq & -\mathcal{E}(w(t))+C_{*}\|w(t)\|_{L^{2}(v)}^{2}+C_{*}\left\|g(t-\delta)-g\left(t-\delta^{\prime}\right)\right\|_{L^{2}(\mu)}^{2} \\
& +C_{*}\left|\delta-\delta^{\prime}\right|^{2-d_{s}}\left\|g\left(t-\delta^{\prime}\right)\right\|_{L^{2}(\mu)}^{2} .
\end{aligned}
$$

It follows from the above inequality and Grönwall's inequality that

$$
\begin{aligned}
\|w\|_{L^{\infty}\left(0, T ; L^{2}(\nu)\right)}+\|w\|_{L^{2}(0, T ; \mathcal{F})} \leq & C_{*}\left[\left|\delta-\delta^{\prime}\right|^{2-d_{s}}\|g\|_{L^{2}\left(0, T ; L^{2}(\mu)\right)}\right. \\
& \left.+\int_{0}^{T}\left\|g(t-\delta)-g\left(t-\delta^{\prime}\right)\right\|_{L^{2}(\mu)}^{2} d t\right] .
\end{aligned}
$$

Therefore, $\left\{u_{\delta}\right\}$ is a Cauchy sequence with respect to the norm $\|\cdot\|_{L^{\infty}\left(0, T ; L^{2}(v)\right)}+\| \cdot$ $\|_{L^{2}(0, T ; \mathcal{F})}$, which proves the convergence of (4.6). Moreover, the desired estimates for $u$ follows readily from the similar estimates for $u_{\delta}$.

Definition 4.10 By virtue of Lemma 4.9, the convolution $\int_{0}^{t} P_{t-s}(g(s) \mu) d s$ can be defined to be the limit in (4.6).

Lemma 4.11 If $g \in L^{\infty}\left(0, T ; L^{2}(\mu)\right)$, then the convolution $u$ defined by (4.6) is jointly continuous in $[0, T] \times \mathbb{S}$. Moreover, for any $0<\theta<\frac{3}{2}\left(1-d_{S} / 2\right)$,

$$
|u(t, x)-u(s, y)| \leq\|g\|_{L^{\infty}\left(0, T ; L^{2}(\mu)\right)}\left[C_{\theta}|t-s|^{\theta}+C_{T} R(x, y)^{1 / 2}\right]
$$

where $C_{\theta}>0$ is a constant depending only on $\theta$, and $C_{T}>0$ one depending only on $T$.

Remark 4.12 The authors believe that $1 / 2$ is the correct Hölder exponent in $x \in \mathbb{S}$ for (4.6) in general, which is suggested by the fact that a generic $u \in \mathcal{F}(\mathbb{S})$ has only 
$\frac{1}{2}$-Hölder continuity [cf. (2.4)]. As a matter of fact, the convolution (4.6) only has mild regularity in general due to the singularity between $v$ and $\mu$ [cf. Remark 4.14.(ii) below].

Proof Let $g(t)=0$ for $t<0$. We first show that

$$
|u(t, x)-u(t, y)| \leq C_{T} R(x, y)^{1 / 2}, \quad x, y \in \mathbb{S},
$$

where $C_{T}>0$ is a constant depending only on $T$. Denote $p_{s, x}(y)=p(s, x, y)$. By the definition of $u(t)$, we have

$$
\begin{aligned}
|u(t, x)-u(t, y)| & =\left|\int_{0}^{t}\left\langle g(t-s), p_{s, x}-p_{s, y}\right\rangle_{\mu} d s\right| \\
& \leq\|g\|_{L^{\infty}\left(0, T, L^{2}(\mu)\right)} \int_{0}^{t}\left\|p_{s, x}-p_{s, y}\right\|_{L^{2}(\mu)} d s .
\end{aligned}
$$

By the Sobolev inequality (3.22),

$$
\left\|p_{s, x}-p_{s, y}\right\|_{L^{2}(\mu)} \leq C \mathcal{E}\left(p_{s, x}-p_{s, y}\right)^{\left(d_{s}-1\right) / 2}\left\|p_{s, x}-p_{s, y}\right\|_{L^{2}(v)}^{2-d_{s}} .
$$

Let $-\mathcal{L}=\int_{0}^{\infty} \lambda d E_{\lambda}$ be the spectral representation. Then

$$
\begin{aligned}
\mathcal{E}\left(p_{s, x}-p_{s, y}\right) & =\mathcal{E}\left(P_{s / 2}\left(p_{s / 2, x}-p_{s / 2, y}\right)\right) \\
& =\int_{0}^{\infty} \lambda e^{-\lambda s} d\left\|E_{\lambda}\left(p_{s / 2, x}-p_{s / 2, y}\right)\right\|_{L^{2}(v)}^{2} \\
& \leq s^{-1}\left\|p_{s / 2, x}-p_{s / 2, y}\right\|_{L^{2}(v)}^{2},
\end{aligned}
$$

Therefore

$$
\left\|p_{s, x}-p_{s, y}\right\|_{L^{2}(\mu)} \leq C s^{-\left(d_{s}-1\right) / 2}\left\|p_{s / 2, x}-p_{s / 2, y}\right\|_{L^{2}(v)}^{d_{s}-1}\left\|p_{s, x}-p_{s, y}\right\|_{L^{2}(v)}^{2-d_{s}},
$$

By the inequality above and Hölder's inequality,

$$
\begin{aligned}
\int_{0}^{t}\left\|p_{s, x}-p_{s, y}\right\|_{L^{2}(\mu)} d s \leq & C\left(\int_{0}^{t} s^{1-d_{s}} d s\right)^{1 / 2}\left(\int_{0}^{t}\left\|p_{s / 2, x}-p_{s / 2, y}\right\|_{L^{2}(v)}^{2} d s\right)^{\left(d_{s}-1\right) / 2} \\
& \times\left(\int_{0}^{t}\left\|p_{s, x}-p_{s, y}\right\|_{L^{2}(v)}^{2} d s\right)^{1-d_{s} / 2} \\
\leq & C_{T}\left(\int_{0}^{t}\left\|p_{s / 2, x}-p_{s / 2, y}\right\|_{L^{2}(v)}^{2} d s\right)^{1 / 2}
\end{aligned}
$$

where we have used the fact that $p_{s, x}-p_{s, y}=P_{s / 2}\left(p_{s / 2, x}-p_{s / 2, y}\right)$ and the $L^{2}(v)$ contractivity of $P_{S / 2}$ for the last inequality. 
Let $\rho_{\alpha}(\cdot, \cdot)$ be the $\alpha$-resolvent kernel. By the Chapman-Kolmogorov equation,

$$
\begin{aligned}
\int_{0}^{t}\left\|p_{s / 2, x}-p_{s / 2, y}\right\|_{L^{2}(v)}^{2} d s & \leq e^{\alpha t} \int_{0}^{\infty} e^{-\alpha s}\left\|p_{s / 2, x}-p_{s / 2, y}\right\|_{L^{2}(v)}^{2} d s \\
& =e^{\alpha t} \int_{0}^{\infty} e^{-\alpha s}[p(s, x, x)-2 p(s, x, y)+p(s, y, y)] d s \\
& =e^{\alpha t}\left[\rho_{\alpha}(x, x)-2 \rho_{\alpha}(x, y)+\rho_{\alpha}(y, y)\right],
\end{aligned}
$$

which, together with Lemma 4.2, implies that

$$
\int_{0}^{t}\left\|p_{s / 2, x}-p_{s / 2, y}\right\|_{L^{2}(v)}^{2} d s \leq C_{\alpha} R(x, y)
$$

Therefore, we deduce that

$$
\int_{0}^{t}\left\|p_{s, x}-p_{s, y}\right\|_{L^{2}(\mu)} d s \leq C_{T} R(x, y)^{1 / 2}
$$

Now the Hölder continuity (4.9) follows readily from (4.10) and (4.12).

Next, we turn to the Hölder continuity of $u(t, x)$ in $t$. Let $t \geq 0, \delta>0$. By the definition of $u$,

$$
\begin{aligned}
u(t+\delta, x)-u(t, x)= & \int_{t}^{t+\delta} P_{t+\delta-s}(g(s) \mu)(x) d s \\
& +\int_{0}^{t}\left[P_{t+\delta-s}(g(s) \mu)(x)-P_{t-s}(g(s) \mu)(x)\right] d s \\
= & I_{1}(\delta)+I_{2}(\delta) .
\end{aligned}
$$

For $I_{1}(\delta)$, in the same way as (4.10), we have

$$
\left|I_{1}(\delta)\right|=\left|\int_{0}^{\delta} P_{S}(g(t-s+\delta) \mu)(x) d s\right| \leq\|g\|_{L^{\infty}\left(0, T ; L^{2}(\mu)\right)} \int_{0}^{\delta}\left\|p_{s, x}\right\|_{L^{2}(\mu)} d s
$$

By the Sobolev inequality (3.22),

$$
\left\|p_{s, x}\right\|_{L^{2}(\mu)} \leq C \mathcal{E}\left(p_{s, x}\right)^{\left(d_{s}-1\right) / 2}\left\|p_{s, x}\right\|_{L^{2}(v)}^{2-d_{s}}
$$

It follows from an argument similar to (4.11) that $\mathcal{E}\left(p_{s, x}\right) \leq s^{-1}\left\|p_{s / 2, x}\right\|_{L^{2}(v)}^{2}$. Therefore,

$$
\left\|p_{s, x}\right\|_{L^{2}(\mu)} \leq C s^{-\left(d_{s}-1\right) / 2}\left\|p_{s / 2, x}\right\|_{L^{2}(v)}^{d_{s}-1}\left\|p_{s, x}\right\|_{L^{2}(v)}^{2-d_{s}} .
$$


Using the Chapman-Kolmogorov equation and the estimate $p(t, x, y) \leq C_{*} t^{-d_{s} / 2}$, we deduce from the above inequality that

$$
\begin{aligned}
\int_{0}^{\delta}\left\|p_{s, x}\right\|_{L^{2}(\mu)} d s & \leq C \int_{0}^{\delta} s^{-\left(d_{s}-1\right) / 2} p(s, x, x)^{\left(d_{s}-1\right) / 2} p(2 s, x, x)^{1-d_{s} / 2} d s \\
& \leq C_{*} \int_{0}^{\delta} s^{-3 d_{s} / 4+1 / 2} d s=C_{*} \delta^{\frac{3}{2}\left(1-d_{s} / 2\right)}
\end{aligned}
$$

Thus

$$
\left|I_{1}(\delta)\right| \leq C_{*}\|g\|_{L^{\infty}\left(0, T ; L^{2}(\mu)\right)} \delta^{\frac{3}{2}\left(1-d_{s} / 2\right)} .
$$

For $I_{2}(\delta)$, by the same argument as in the estimate of $\left|I_{1}(\delta)\right|$, we have

$$
\begin{aligned}
\left|I_{2}(\delta)\right| \leq & \|g\|_{L^{\infty}\left(0, T ; L^{2}(\mu)\right)} \int_{0}^{t}\left\|p_{s+\delta, x}-p_{s, x}\right\|_{L^{2}(\mu)} d s \\
\leq & C_{*}\|g\|_{L^{\infty}\left(0, T ; L^{2}(\mu)\right)} \int_{0}^{t}\left\|p_{s / 2+\delta, x}-p_{s / 2, x}\right\|_{L^{2}(v)}^{d_{s}-1} \| p_{s+\delta, x} \\
& \quad-p_{s, x} \|_{L^{2}(v)}^{2-d_{s}} \frac{d s}{s^{\left(d_{s}-1\right) / 2}} \\
\leq & C_{*}\|g\|_{L^{\infty}\left(0, T ; L^{2}(\mu)\right)} \int_{0}^{t}\left\|p_{s / 2+\delta, x}-p_{s / 2, x}\right\|_{L^{2}(v)} \frac{d s}{s^{\left(d_{s}-1\right) / 2}} .
\end{aligned}
$$

For any $\theta \in[0,1]$, by the spectral representation,

$$
\begin{aligned}
\left\|p_{s / 2+\delta, x}-p_{s / 2, x}\right\|_{L^{2}(v)}^{2} & =\left\|\left(P_{s / 4+\delta}-P_{s / 4}\right) p_{s / 4, x}\right\|_{L^{2}(v)}^{2} \\
& =\int_{0}^{\infty}\left(1-e^{-\delta \lambda}\right)^{2} e^{-s \lambda / 2} d\left\|E_{\lambda} p_{s / 4, x}\right\|_{L^{2}(v)}^{2} \\
& \leq \delta^{2 \theta} \int_{0}^{\infty} \lambda^{2 \theta} e^{-s \lambda / 2} d\left\|E_{\lambda} p_{s / 4, x}\right\|_{L^{2}(v)}^{2} \\
& \leq C_{*}(\delta / s)^{2 \theta}\left\|p_{s / 4, x}\right\|_{L^{2}(v)}^{2}=C_{*}(\delta / s)^{2 \theta} p(s / 2, x, x) \\
& \leq C_{*} \delta^{2 \theta} s^{-2 \theta-d_{s} / 2},
\end{aligned}
$$

which, together with (4.14), implies that

$$
\left|I_{2}(\delta)\right| \leq C_{*}\|g\|_{L^{\infty}\left(0, T ; L^{2}(\mu)\right)} \delta^{\theta} \int_{0}^{t} s^{\frac{3}{2}\left(1-d_{s} / 2\right)-\theta} \frac{d s}{s} .
$$

Therefore, for any $\theta<\frac{3}{2}\left(1-d_{s} / 2\right)$, the estimate

$$
\left|I_{2}(\delta)\right| \leq C_{\theta}\|g\|_{L^{\infty}\left(0, T ; L^{2}(\mu)\right)} \delta^{\theta},
$$

is valid. Combining (4.13) and (4.15), we deduce that

$$
|u(t, x)-u(s, x)| \leq C_{\theta}\|g\|_{L^{\infty}\left(0, T ; L^{2}(\mu)\right)}|t-s|^{\theta},
$$


for all $0<\theta<\frac{3}{2}\left(1-d_{s} / 2\right)$.

Now the joint Hölder continuity (4.8) follows readily from (4.9) and (4.16).

Definition 4.13 A function $u$ is called a weak solution to the PDE (4.1) if:

(WS.1) $u \in L^{2}\left(0, T ; \mathcal{F}\left(\mathbb{S} \backslash V_{0}\right)\right)$ and $u$ has a weak derivative $\partial_{t} u$ in $L^{2}\left(0, T ; \mathcal{F}^{-1}(\mathbb{S})\right)$; (WS.2) For any $v \in \mathcal{F}\left(\mathbb{S} \backslash \mathrm{V}_{0}\right)$,

$$
\left\langle\partial_{t} u(t), v\right\rangle_{v}=-\mathcal{E}(u(t), v)+\langle f(t, u(t), \nabla u(t)), v\rangle_{\mu}, \quad \text { a.e. } t \in[0, T]
$$

(WS.3) $\lim _{t \rightarrow 0} u(t)=\psi$ in $L^{2}(v)$.

Remark 4.14 (i) The term $\langle f(t, u(t), \nabla u(t)), v\rangle_{\mu}$ in (WS.2) is legitimate since $\nabla u$ is $\mu$-a.e. defined and $u \in \mathcal{F}(\mathbb{S}) \subseteq C(\mathbb{S})$.

(ii) Notice that, in general, the Eq. (4.1) does not admit a solution $u$ such that $u(t) \in$ $\operatorname{Dom}(\mathcal{L})$ and $\partial_{t} u(t) \in L^{2}(v)$ for a.e. $t \in[0, T]$. Otherwise, the functional $v \mapsto$ $\langle f(t, u, \nabla u), v\rangle_{\mu}$ will be $L^{2}(v)$-bounded, which contradicts with the singularity between $v$ and $\mu$. Therefore, solutions to non-linear parabolic PDEs on $\mathbb{S}$ can only have mild regularity in general. This is a remarkable feature of non-linear PDEs on $\mathbb{S}$, which suggests a significant distinction between the PDE theory on Euclidean spaces and that on fractals.

(iii) We shall show that if $u$ is a weak solution to (4.1) then $u \in C((0, T] \times \mathbb{S})$ (see Theorem 4.16 below). Therefore, Definition 4.13 coincides with the definition of solutions in [27, Definition 3.17]. The joint continuity of solutions is needed for the validity of the Feynman-Kac representation given by [27, Theorem 3.19], which will be crucial in the study of the Burgers equations on $\mathbb{S}$ (see Sect. 5).

Proposition 4.15 Suppose that $g \in L^{2}\left(0, T ; L^{2}(\mu)\right)$. Then the initial and boundary problem to the PDE

$$
\left\{\begin{array}{c}
\partial_{t} u d v=\mathcal{L} u d v+g(t, x) d \mu, \quad \text { in }(0, T] \times\left(\mathbb{S} \backslash \mathrm{V}_{0}\right), \\
u=0 \text { on }(0, T] \times \mathrm{V}_{0}, \quad u(0)=\psi
\end{array}\right.
$$

admits a unique weak solution u given by

$$
u(t)=P_{t} \psi+\int_{0}^{t} P_{t-s}(g(s) \mu) d s, \quad t \in[0, T] .
$$

Moreover

$$
\begin{aligned}
& \|u\|_{L^{\infty}\left(0, T ; L^{2}(v)\right)}+\|u\|_{L^{2}(0, T ; \mathcal{F})}+\left\|\partial_{t} u\right\|_{L^{2}(0, T ; \mathcal{F}-1)} \\
& \quad \leq C_{*}\left(\|\psi\|_{L^{2}(v)}+\|g\|_{L^{2}\left(0, T ; L^{2}(\mu)\right)}\right) .
\end{aligned}
$$

Proof Clearly, we only need to prove for the case when $\psi=0$. Let $u_{\delta}$ be the truncated convolution defined by (4.3), and let $u$ be the convolution given by (4.6). For any $v \in \mathcal{F}\left(\mathbb{S} \backslash \mathrm{V}_{0}\right)$, by (4.4),

$$
\left\langle\partial_{t} u_{\delta}(t), v\right\rangle_{v}=-\mathcal{E}\left(u_{\delta}(t), v\right)+\left\langle P_{\delta}(g(t-\delta) \mu), v\right\rangle_{v}
$$




$$
=-\mathcal{E}\left(u_{\delta}(t), v\right)+\left\langle g(t-\delta), P_{\delta} v\right\rangle_{\mu}, \text { a.e. } t \in(0, T] \text {. }
$$

Since $\lim _{\delta \rightarrow 0} P_{\delta} v=v$ uniformly, by considering a subsequence if necessary and setting $\delta \rightarrow 0$, we deduce that

$$
\left\langle\partial_{t} u(t), v\right\rangle_{v}=-\mathcal{E}(u(t), v)+\langle g(t), v\rangle_{\mu}, \quad \text { a.e. } t \in(0, T]
$$

Therefore, $u$ is a weak solution to (4.17).

The estimate (4.18) follows readily from Lemma 4.9, and the uniqueness of solutions is an immediate consequence of (4.18).

We are now in a position to state and give the proof of the main result of this section.

Theorem 4.16 Suppose that (A.1) and (A.2) hold. Then (4.1) admits a unique weak solution $u$ satisfying the following estimate

$$
\begin{gathered}
\|u\|_{L^{\infty}\left(0, T ; L^{2}(v)\right)}+\|u\|_{L^{2}(0, T ; \mathcal{F})}+\left\|\partial_{t} u\right\|_{L^{2}(0, T ; \mathcal{F}-1)} \\
\leq C_{K, T}\left(\|\psi\|_{L^{2}(v)}+\|f(\cdot, 0,0)\|_{L^{2}\left(0, T ; L^{2}(\mu)\right)}\right)
\end{gathered}
$$

where $C_{K, T}>0$ is a constant depending only on $T$ and the Lipschitz constant $K$ in (A.1). Moreover, if $\tilde{u}$ is the weak solution to (4.1) with initial value $\tilde{\psi} \in L^{2}(v)$, then

$$
\begin{aligned}
& \|u-\tilde{u}\|_{L^{\infty}\left(0, T ; L^{2}(v)\right)}+\|u-\tilde{u}\|_{L^{2}(0, T ; \mathcal{F})} \\
& \quad+\left\|\partial_{t} u-\partial_{t} \tilde{u}\right\|_{L^{2}\left(0, T ; \mathcal{F}^{-1}\right)} \leq C_{K, T}\|\psi-\tilde{\psi}\|_{L^{2}(v)} .
\end{aligned}
$$

Suppose, in addition, that $\psi \in \mathcal{F}\left(\mathbb{S} \backslash \mathrm{V}_{0}\right)$ and $f(\cdot, 0,0)=0$. Then

$$
\|u\|_{L^{\infty}(0, T ; \mathcal{F})} \leq C_{K, T} \mathcal{E}(\psi)^{1 / 2} .
$$

Moreover, $u(t, x)$ is jointly continuous in $(0, T] \times \mathbb{S}$, with $\theta$-Hölder continuity in $t \in(0, T]$ for any $\theta<\frac{3}{2}\left(1-d_{S} / 2\right)$ and $\frac{1}{2}$-Hölder continuity in $x \in \mathbb{S}$ with respect to the resistance metric.

Proof We first prove the existence. Let $u^{0}(t)=P_{t} \psi, t \in[0, T]$. By Proposition 4.15, we may define a sequence $\left\{u^{n}\right\}_{n \in \mathbb{N}_{+}}$in $L^{2}\left(0, T ; \mathcal{F}\left(\mathbb{S} \backslash \mathrm{V}_{0}\right)\right)$ inductively by

$$
\left\{\begin{aligned}
\partial_{t} u^{n} d v & =\mathcal{L} u^{n} d v+f^{n-1}(t) d \mu \\
\left.u^{n}\right|_{\mathrm{V}_{0}} & =0, \quad u^{n}(0)=\psi
\end{aligned}\right.
$$

where $f^{n-1}(t, x)=f\left(t, x, u^{n-1}(t, x), \nabla u^{n-1}(t, x)\right)$. By Proposition 4.15, $u^{n} \in$ $L^{2}\left(0, T ; \mathcal{F}\left(\mathbb{S} \backslash \mathrm{V}_{0}\right)\right), \partial_{t} u^{n} \in L^{2}\left(0, T ; \mathcal{F}^{-1}(\mathbb{S})\right)$. Denote $w^{n}=u^{n}-u^{n-1}, n \in \mathbb{N}_{+}$. By (4.22), $w^{n+1}, n \in \mathbb{N}_{+}$is the solution to

$$
\left\{\begin{aligned}
\partial_{t} w^{n+1} d v & =\mathcal{L} w^{n+1} d v+\left[f^{n}(t)-f^{n-1}(t)\right] d \mu \\
w^{n+1} \mid \mathrm{V}_{0} & =0, \quad w^{n+1}(0)=0
\end{aligned}\right.
$$


For any $\epsilon \in(0,1)$, by Lemma 4.7.(b), testing (4.23) against $w^{n+1}(t)$ gives that

$$
\begin{aligned}
\frac{1}{2} \frac{d}{d t}\left\|w^{n+1}(t)\right\|_{L^{2}(v)}^{2} \leq & -\mathcal{E}\left(w^{n+1}(t)\right)+\frac{1}{32} \mathcal{E}\left(w^{n}(t)\right)+\epsilon^{2}\left\|w^{n}(t)\right\|_{L^{2}(\mu)}^{2} \\
& \left.+C_{K}\left\|w^{n+1}(t)\right\|_{L^{2}(\mu)}^{2}\right)
\end{aligned}
$$

where, and in the rest of the proof, $C_{K}>0$ denotes a generic constant depending only on $K$ which may vary on different occasions. Since $w^{n} \mid \mathrm{v}_{0}=0$, by (2.5), we have $\left\|w^{n}(t)\right\|_{L^{2}(\mu)}^{2} \leq C_{*}^{2} \mathcal{E}\left(w^{n}(t)\right)$. Moreover, by Corollary 3.18,

$$
\begin{aligned}
\frac{1}{2} \frac{d}{d t}\left\|w^{n+1}(t)\right\|_{L^{2}(v)}^{2} \leq & -\left(1-C_{K} \epsilon^{2}\right) \mathcal{E}\left(w^{n+1}(t)\right) \\
& +\left(\frac{1}{32}+C_{*}^{2} \epsilon^{2}\right) \mathcal{E}\left(w^{n}(t)\right)+C_{K} C_{\epsilon}^{2}\left\|w^{n+1}(t)\right\|_{L^{2}(v)}^{2},
\end{aligned}
$$

where $C_{\epsilon}>0$ is a constant depending only on $\epsilon$. By choosing $\epsilon>0$ sufficiently small, we have that

$$
\frac{d}{d t}\left\|w^{n+1}(t)\right\|_{L^{2}(v)}^{2} \leq-\mathcal{E}\left(w^{n+1}(t)\right)+\frac{1}{8} \mathcal{E}\left(w^{n}(t)\right)+C_{K}\left\|w^{n+1}(t)\right\|_{L^{2}(v)}^{2},
$$

By the above and Grönwall's inequality,

$$
\left\|w^{n+1}(t)\right\|_{L^{2}(v)}^{2}+\int_{0}^{t} e^{C_{K}(t-s)} \mathcal{E}\left(w^{n+1}(s)\right) d s \leq \frac{1}{8} \int_{0}^{t} e^{C_{K}(t-s)} \mathcal{E}\left(w^{n}(s)\right) d s,
$$

which implies that

$$
\begin{aligned}
& \left\|u^{m}-u^{m-1}\right\|_{L^{\infty}\left(0, T ; L^{2}(v)\right)}+\left(\int_{0}^{T} e^{C_{K}(T-s)} \mathcal{E}\left(u^{m}(s)-u^{m-1}(s)\right) d s\right)^{1 / 2} \\
& \leq 2^{-m+n}\left[\left\|u^{n}-u^{n-1}\right\|_{L^{\infty}\left(0, T ; L^{2}(v)\right)}\right. \\
& \left.\quad+\left(\int_{0}^{T} e^{C_{K}(T-s)} \mathcal{E}\left(u^{n}(s)-u^{n-1}(s)\right) d s\right)^{1 / 2}\right]
\end{aligned}
$$

for all $m \geq n$, and that

$$
\begin{aligned}
& \left\|u^{n}\right\|_{L^{\infty}\left(0, T ; L^{2}(v)\right)}+\left(\int_{0}^{T} e^{C_{K}(T-s)} \mathcal{E}\left(u^{n}(s)\right) d s\right)^{1 / 2} \\
& \leq\left\|u^{1}\right\|_{L^{\infty}\left(0, T ; L^{2}(v)\right)}+\left(\int_{0}^{T} e^{C_{K}(T-s)} \mathcal{E}\left(u^{1}(s)\right) d s\right)^{1 / 2} .
\end{aligned}
$$


Moreover, by Proposition 4.15, we have

$$
\begin{aligned}
& \left\|u^{1}\right\|_{L^{\infty}\left(0, T ; L^{2}(v)\right)}+\left(\int_{0}^{T} e^{C_{K}(T-s)} \mathcal{E}\left(u^{1}(s)\right) d s\right)^{1 / 2} \\
& \quad \leq C_{*} e^{C_{K} T}\left(\|\psi\|_{L^{2}(v)}+\left\|f\left(\cdot, u^{0}, \nabla u^{0}\right)\right\|_{L^{2}\left(0, T ; L^{2}(\mu)\right)}\right) \\
& \quad \leq C_{*} e^{C_{K} T}\left(\|\psi\|_{L^{2}(v)}+\left\|u^{0}\right\|_{L^{2}(0, T ; \mathcal{F})}+\|f(\cdot, 0,0)\|_{L^{2}\left(0, T ; L^{2}(\mu)\right)}\right) .
\end{aligned}
$$

Let $-\mathcal{L}=\int_{0}^{\infty} \lambda d E_{\lambda}$ be the spectral decomposition. Then

$$
\begin{aligned}
\left\|u^{0}\right\|_{L^{2}(0, T ; \mathcal{F})}^{2} & \leq T\|\psi\|_{L^{2}(v)}^{2}+\int_{0}^{T} \mathcal{E}\left(P_{t} \psi\right) d t \\
& =T\|\psi\|_{L^{2}(v)}^{2}+\int_{0}^{T} \int_{0}^{\infty} \lambda e^{-2 t \lambda} d\left\|E_{\lambda} \psi\right\|_{L^{2}(v)}^{2} d t \\
& =T\|\psi\|_{L^{2}(v)}^{2}+\frac{1}{2} \int_{0}^{\infty}\left(1-e^{-2 T \lambda}\right) d\left\|E_{\lambda} \psi\right\|_{L^{2}(v)}^{2} \\
& \leq(T+1)\|\psi\|_{L^{2}(v)}^{2} .
\end{aligned}
$$

Therefore, we obtain that

$$
\begin{aligned}
& \left\|u^{n}\right\|_{L^{\infty}\left(0, T ; L^{2}(v)\right)}+\left(\int_{0}^{T} e^{C_{K}(T-s)} \mathcal{E}\left(u^{n}(s)\right) d s\right)^{1 / 2} \\
& \quad \leq C_{*} e^{C_{K} T}\left(\|\psi\|_{L^{2}(v)}+\|f(\cdot, 0,0)\|_{L^{2}\left(0, T ; L^{2}(\mu)\right)}\right), \quad n \in \mathbb{N}_{+} .
\end{aligned}
$$

Furthermore, by (4.22), $u^{m}-u^{n}$ is the solution to

$$
\left\{\begin{array}{c}
\partial_{t}\left(u^{m}-u^{n}\right) d v=\mathcal{L}\left(u^{m}-u^{n}\right) d v+\left[f^{m-1}(t)-f^{n-1}(t)\right] d \mu, \\
\left.\left(u^{m}-u^{n}\right)\right|_{\mathrm{V}_{0}}=0, \quad\left(u^{m}-u^{n}\right)(0)=0 .
\end{array}\right.
$$

For any $v \in \mathcal{F}\left(\mathbb{S} \backslash \mathrm{V}_{0}\right)$, by the above equation,

$$
\left|\left\langle\partial_{t}\left(u^{m}-u^{n}\right), v\right\rangle_{\nu}\right| \leq\left[\mathcal{E}\left(u^{m}-u^{n}\right)^{1 / 2}+C_{K} \mathcal{E}\left(u^{m-1}-u^{n-1}\right)^{1 / 2}\right] \mathcal{E}(v)^{1 / 2},
$$

which implies that

$$
\left\|\partial_{t}\left(u^{m}-u^{n}\right)\right\|_{\mathcal{F}^{-1}} \leq \mathcal{E}\left(u^{m}-u^{n}\right)^{1 / 2}+C_{K} \mathcal{E}\left(u^{m-1}-u^{n-1}\right)^{1 / 2}
$$

By (4.26) and (4.27), we see that

$\left\|\partial_{t}\left(u^{m}-u^{n}\right)\right\|_{L^{2}\left(0, T ; \mathcal{F}^{-1}\right)} \leq C_{*} e^{C_{K} T} 2^{-(m-n)}\left(\|\psi\|_{L^{2}(v)}+\|f(\cdot, 0,0)\|_{L^{2}\left(0, T ; L^{2}(\mu)\right)}\right)$.

Therefore, $\left\{u^{n}\right\}$ is a $\|\cdot\|_{*}$-Cauchy sequence satisfying

$$
\left\|u^{n}\right\|_{*} \leq C_{*} e^{C_{K} T}\left(\|\psi\|_{L^{2}(v)}+\|f(\cdot, 0,0)\|_{L^{2}\left(0, T ; L^{2}(\mu)\right)}\right), \quad n \in \mathbb{N}_{+},
$$


where

$$
\|u\|_{*}=\|u\|_{L^{\infty}\left(0, T ; L^{2}(v)\right)}+\|u\|_{L^{2}(0, T ; \mathcal{F})}+\left\|\partial_{t} u\right\|_{L^{2}(0, T ; \mathcal{F}-1)} .
$$

Therefore, there exists a $u \in L^{2}\left(0, T ; \mathcal{F}\left(\mathbb{S} \backslash \mathrm{V}_{0}\right)\right)$ such that $\lim _{n \rightarrow \infty}\left\|u^{n}-u\right\|_{*}=0$. It is clear that $u$ is a weak solution to (4.1), and the estimate (4.19) holds as $\mathcal{E}^{1 / 2}(\cdot)$ and $\|\cdot\|_{\mathcal{F}}$ are equivalent on $\mathcal{F}\left(\mathbb{S} \backslash \mathrm{V}_{0}\right)$. This proves the existence.

Suppose that $\tilde{u}$ is a weak solution to (4.1) with initial value $\tilde{\psi}$. By an argument similar to (4.24) and (4.28), it can be shown that

$$
\frac{d}{d t}\|u(t)-\tilde{u}(t)\|_{L^{2}(v)}^{2} \leq-\frac{1}{2} \mathcal{E}(u(t)-\tilde{u}(t))+C_{K}\|u(t)-\tilde{u}(t)\|_{L^{2}(v)}^{2},
$$

and that

$$
\left\|\partial_{t}(u-\tilde{u})\right\|_{\mathcal{F}^{-1}} \leq C_{K} \mathcal{E}(u-\tilde{u})^{1 / 2}
$$

The estimate (4.20) follows readily from the above two inequalities. The uniqueness of solutions is now an immediate consequence of (4.20).

Suppose, in addition, that $\psi \in \mathcal{F}\left(\mathbb{S} \backslash \mathrm{V}_{0}\right)$ and $f(\cdot, 0,0)=0$. Then (4.25) also holds for $n=0$ with $u^{-1}=0$. Therefore,

$$
\int_{0}^{T} \mathcal{E}\left(u^{m}(t)\right) d t \leq e^{C_{K} T} \int_{0}^{T} \mathcal{E}\left(u^{0}(t)\right) d t=e^{C_{K} T} \int_{0}^{T} \mathcal{E}\left(P_{t} \psi\right) d t \leq T e^{C_{K} T} \mathcal{E}(\psi),
$$

which implies that

$$
\int_{0}^{T} \mathcal{E}(u(t)) d t \leq T e^{C_{K} T} \mathcal{E}(\psi) .
$$

Now for any $\delta \in(0, T), u$ is the solution to

$$
\left\{\begin{aligned}
\partial_{t} u d v & =\mathcal{L} u d v+f(t, x, u, \nabla u) d \mu, \quad t \in\left(t_{0}, t_{0}+\delta\right] \\
\left.u\right|_{\mathrm{V}_{0}} & =0,\left.\quad u\right|_{t=t_{0}}=u\left(t_{0}\right)
\end{aligned}\right.
$$

Applying (4.29) to the above PDE and using $\|u\|_{L^{2}(v)}^{2} \leq C_{*} \mathcal{E}(u)$ gives that

$$
\frac{1}{\delta} \int_{t_{0}}^{t_{0}+\delta} \mathcal{E}(u(t)) d t \leq e^{C_{K} \delta} \mathcal{E}\left(u\left(t_{0}\right)\right), \text { a.e. } t_{0} \in[0, T-\delta] \text { and any } \delta>0 .
$$

We claim that (4.30) implies (4.21). We first show the following lemma.

Lemma Let $h(t)$ be a locally integrable function on $[0, \infty)$ satisfying

$$
\frac{1}{\delta} \int_{t}^{t+\delta} h(s) d s \leq L \delta+h(t), \text { a.e. } t \in[0, \infty) \text { and any } \delta>0
$$

for some constant $L>0$. Then $h(t)-h(s) \leq 6 L(t-s)$, a.e. $0<s \leq t<\infty$. 
To prove the lemma, suppose first that $h$ is differentiable on $(0, \infty)$. Suppose the contrary that $h(t)-h(s)>3 L(t-s)$ for some $0<s<t<\infty$. Then there exists an $t_{0} \in(s, t)$ such that $h^{\prime}\left(t_{0}\right)>3 L$. Moreover, $h(r)-h\left(t_{0}\right)>3 L\left(r-t_{0}\right), r \in\left[t_{0}, t_{0}+\delta\right]$ for $\delta>0$ sufficiently small. This implies that $\frac{1}{\delta} \int_{t_{0}}^{t_{0}+\delta} h(r) d r>3 L \delta / 2+h\left(t_{0}\right)$, which contradicts (4.31). This proves the lemma for differentiable functions $h$.

For general $h$, let $h_{\epsilon}(t)=\frac{1}{\epsilon} \int_{t}^{t+\epsilon} h(s) d s, \epsilon>0$. Then $h_{\epsilon}$ is differentiable and satisfies (4.31) with $L$ replaced by $2 L$. The above case gives that $h_{\epsilon}(t)-h_{\epsilon}(s) \leq$ $6 L(t-s)$. It remains to apply the Lebesgue differentiation theorem to complete the proof of the lemma.

Now by (4.30) and Jensen's inequality, the function $h(t)=\log [\mathcal{E}(u(t))]$ satisfies (4.31) with $L=C_{K}$. It follows from the previous lemma that

$$
\mathcal{E}(u(t)) \leq e^{C_{K}(t-s)} \mathcal{E}(u(s)) \text {, a.e. } 0<s \leq t \leq T .
$$

Using the above inequality and (4.29) again, we deduce that

$$
\mathcal{E}(u(t)) \leq \frac{1}{t} \int_{0}^{t} e^{C_{K}(t-s)} \mathcal{E}(u(s)) d s \leq e^{C_{K} t} \mathcal{E}(\psi), \text { a.e. } t \in(0, T],
$$

which implies (4.21).

We now prove the joint Hölder continuity. Let $g(t, x)=f(t, x, u(t, x), \nabla u(t, x))$. Then $u$ is the solution to the PDE

$$
\partial_{t} u d v=\mathcal{L} u d v+g(t) d \mu .
$$

By Proposition 4.15,

$$
u(t)=P_{t} \psi+\int_{0}^{t} P_{t-s}(g(s) \mu) d s .
$$

By (4.21) and the Sobolev inequality (3.22), it is easily seen that

$$
\|g\|_{L^{\infty}\left(0, T ; L^{2}(\mu)\right)}<\infty .
$$

We now can apply Lemma 4.11 and Proposition 4.15 and to deduce the desired joint Hölder continuity.

\section{The Burgers equations}

As an application of Theorem 4.16 and the Feynman-Kac representation for (backward) parabolic PDEs on $\mathbb{S}$ in [27, Theorem 3.19], we study the initial-boundary value problem for the following analogue on $\mathbb{S}$ of the Burgers equations on $\mathbb{R}$

$$
\left\{\begin{array}{c}
\partial_{t} u d v=\mathcal{L} u d v+u \nabla u d \mu, \quad \text { in }(0, T] \times\left(\mathbb{S} \backslash \mathrm{V}_{0}\right), \\
u=0 \text { on }(0, T] \times \mathrm{V}_{0}, \quad u(0)=\psi,
\end{array}\right.
$$


where $\psi \in \mathcal{F}\left(\mathbb{S} \backslash \mathrm{V}_{0}\right)$. We shall prove the existence and uniqueness of solutions to the Eq. (5.1), and derive the regularity of the solutions.

Remark 5.1 We would like to point out a difference between the Burgers equations on $\mathbb{S}$ and those on $\mathbb{R}$. The Burgers equations on $\mathbb{R}$ can be exactly solved with an explicit formula for the solutions via the Cole-Hopf transformation, and properties of solutions can be derived using the explicit formula. However, this Cole-Hopf type of transformation is not available on $\mathbb{S}$. The Cole-Hopf transformation reduces the Burgers equation on $\mathbb{R}$ for $u$ to a heat equation for $-\nabla(\log u)$. In contrast, on $\mathbb{S}$, the formal expression $\mathcal{L}[\nabla(\log u)]$ is not well-defined, since the gradient $\nabla(\log u)$ is only $\mu$-a.e. defined and therefore $\nabla(\log u) \notin \mathcal{F}(\mathbb{S})$ due to the singularity between $\nu$ and $\mu$. Hence, different approaches must be employed for the study of (5.1).

Let us start with the Feynman-Kac representation for solutions to parabolic PDEs on $\mathbb{S}$. Let $\left\{X_{t}\right\}_{t \geq 0}$ and $\left\{W_{t}\right\}_{t \geq 0}$ be Brownian motion and the representing martingale on $\mathbb{S}$ respectively, i.e. $\left\{X_{t}\right\}_{t \geq 0}$ is the diffusion process associated with the form $(\mathcal{E}, \mathcal{F}(\mathbb{S}))$, and $\left\{W_{t}\right\}_{t \geq 0}$ is the unique martingale additive functional having $\mu$ as its energy measure such that $M_{t}^{[u]}=\int_{0}^{t} \nabla u\left(X_{r}\right) d W_{r}$ for any $u \in \mathcal{F}(\mathbb{S})$, where $M^{[u]}$ is the martingale part of $u\left(X_{t}\right)-u\left(X_{0}\right)$ (cf. [25, Theorem 5.4] and [27, Section 2]). The following result was given in [27, Theorem 3.19], and is an analogue on $\mathbb{S}$ of the representation theorem for semi-linear PDEs on $\mathbb{R}^{d}$ established by Peng in [29]. See [27, Section 3] for the definition of solutions to backward stochastic differential equations (BSDEs) on $\mathbb{S}$.

Theorem 5.2 If the PDE (4.1) admits a weak solution u jointly continuous in $(0, T] \times \mathbb{S}$, then

$$
\left(Y_{t}, Z_{t}\right)=\left(u\left(T-t, X_{t}\right), \nabla u\left(T-t, X_{t}\right)\right)
$$

is the unique solution to the BSDE

$$
\left\{\begin{array}{l}
d Y_{t}=-f\left(T-t, X_{t}, Y_{t}, Z_{t}\right) d\langle W\rangle_{t}+Z_{t} d W_{t}, \quad t \in\left[0, \sigma^{(T)}\right) \\
Y_{\sigma^{(T)}}=\Psi\left(\sigma^{(T)}, X_{\sigma^{(T)}}\right)
\end{array}\right.
$$

on $\left(\Omega, \mathbb{P}_{x}\right)$ for each $x \in \mathbb{S}$, where $\sigma^{(T)}=T \wedge \inf \left\{t>0: X_{t} \in \mathrm{V}_{0}\right\}$, and

$$
\Psi(t, x)=\left\{\begin{array}{cl}
0, & \text { if }(t, x) \in[0, T) \times \mathrm{V}_{0} \\
\psi(x), & \text { if }(t, x) \in\{T\} \times \mathbb{S} \backslash \mathrm{V}_{0}
\end{array}\right.
$$

Moreover, the solution to (4.1) has the representation $u(T, x)=Y_{0}=\mathbb{E}_{x}\left(Y_{0}\right)$ for all $x \in \mathbb{S}$.

Proposition 5.3 The Burgers equation (5.1) admits a unique weak solution u satisfying the maximal principle below

$$
\|u\|_{L^{\infty}\left(0, T ; L^{\infty}\right)} \leq\|\psi\|_{L^{\infty}} .
$$


Moreover,

$$
\|u\|_{L^{\infty}\left(0, T ; L^{2}(v)\right)}+\|u\|_{L^{2}(0, T ; \mathcal{F})}+\left\|\partial_{t} u\right\|_{L^{2}\left(0, T ; \mathcal{F}^{-1}\right)} \leq C,
$$

for some constant $C>0$ depending only on $\|\psi\|_{L^{\infty}}$ and $T$. The solution u is jointly continuous in $(0, T] \times \mathbb{S}$, with $\theta$-Hölder continuity in $t \in(0, T]$ for any $\theta<\frac{3}{2}\left(1-d_{S} / 2\right)$ and $\frac{1}{2}$-Hölder continuity in $x \in \mathbb{S}$ with respect to the resistance metric.

Proof Existence. We define the sequence $\left\{u^{n}\right\}_{n \in \mathbb{N}} \subseteq L^{2}(0, T ; \mathcal{F})$ by induction as follows. Let $u^{0}(t)=P_{t} \psi$. Then $\left\|u^{0}\right\|_{L^{\infty}\left(0, T ; L^{\infty}\right)} \leq\|\psi\|_{L^{\infty}}$. Suppose that $u^{n-1}$ with $\left\|u^{n-1}\right\|_{L^{\infty}\left(0, T ; L^{\infty}\right)} \leq\|\psi\|_{L^{\infty}}$ has been defined. The function $u^{n}$ is defined to be the unique weak solution to the PDE (cf. Theorem 4.16)

$$
\left\{\begin{array}{c}
\partial_{t} u^{n} d v=\mathcal{L} u^{n} d v+u^{n-1} \nabla u^{n} d \mu, \quad \text { in }(0, T] \times\left(\mathbb{S} \backslash \mathrm{V}_{0}\right), \\
u^{n}=0 \text { on }(0, T] \times \mathrm{V}_{0}, \quad u^{n}(0)=\psi
\end{array}\right.
$$

To verify the definition of $\left\{u^{n}\right\}$, we must show that $\left\|u^{n}\right\|_{L^{\infty}\left(0, T ; L^{\infty}\right)} \leq\|\psi\|_{L^{\infty}}$. Without loss of generality, we only need to show that $\left\|u^{n}(T)\right\|_{L^{\infty}} \leq\|\psi\|_{L^{\infty}}$. By Theorem 5.2, $\left(Y_{t}, Z_{t}\right)=\left(u^{n}\left(T-t, X_{t}\right), \nabla u^{n}\left(T-t, X_{t}\right)\right)$ is the unique solution to the BSDE

$$
\left\{\begin{array}{l}
d Y_{t}=-u^{n-1}\left(T-t, X_{t}\right) Z_{t} d\langle W\rangle_{t}+Z_{t} d W_{t}, \quad t \in\left[0, \sigma^{(T)}\right) \\
Y_{\sigma^{(T)}}=\Psi\left(\sigma^{(T)}, X_{\sigma^{(T)}}\right)
\end{array}\right.
$$

where $\sigma^{(T)}=T \wedge \inf \left\{t>0: X_{t} \in \mathrm{V}_{0}\right\}$, and

$$
\Psi(t, x)=\left\{\begin{array}{cl}
0, & \text { if }(t, x) \in[0, T) \times \mathrm{V}_{0} \\
\psi(x), & \text { if }(t, x) \in\{T\} \times \mathbb{S} \backslash \mathrm{V}_{0}
\end{array}\right.
$$

For each $x \in \mathbb{S} \backslash \mathrm{V}_{0}$, we define a measure $\tilde{\mathbb{P}}_{x}$ by

$$
\frac{d \tilde{\mathbb{P}}_{x}}{d \mathbb{P}_{x}}=\exp \left[\int_{0}^{\sigma^{(T)}} u^{n-1}\left(T-r, X_{r}\right) d W_{r}-\frac{1}{2} \int_{0}^{\sigma^{(T)}} u^{n-1}\left(T-r, X_{r}\right)^{2} d\langle W\rangle_{r}\right] .
$$

The measure $\tilde{\mathbb{P}}_{x}$ is a probability measure. In fact, by [27, Corollary 4.3], the quadratic process $\langle W\rangle$ is exponentially integrable, i.e.

$$
\sup _{x \in \mathbb{S}} \mathbb{E}_{x}\left[\exp \left(\beta\langle W\rangle_{T}\right)\right]<\infty
$$

for all $\beta, T>0$. Hence, in view of the uniform boundedness $\left\|u^{n-1}\right\|_{L^{\infty}\left(0, T ; L^{\infty}\right)} \leq$ $\|\psi\|_{L^{\infty}}$, we see that the Novikov condition is satisfied and therefore $\tilde{\mathbb{P}}_{x}$ is a probability martingale measure. By (5.3),

$$
Y_{t}=Y_{0}+\int_{0}^{t} Z_{r} d W_{r}-\left\langle\int Z_{r} d W_{r}, \int u^{n-1}\left(T-r, X_{r}\right) d W_{r}\right\rangle_{t} .
$$


Notice that

$$
\mathbb{E}_{\nu}\left(\int_{0}^{T} Z_{r}^{2} d\langle W\rangle_{r}\right)=\int_{0}^{T}\left\|\nabla u^{n}(T-r)\right\|_{L^{2}(\mu)}^{2} d r \leq\left\|u^{n}\right\|_{L^{2}(0, T ; \mathcal{F})}^{2}<\infty,
$$

which implies that $\mathbb{E}_{x}\left(\int_{0}^{T} Z_{r}^{2} d\langle W\rangle_{r}\right)<\infty$ for $\nu$-a.e. $x \in \mathbb{S}$ and therefore, for all $x \in \mathbb{S}$ in view of the quasi-continuity of the function $x \mapsto \mathbb{E}_{x}\left(\int_{0}^{T} Z_{r}^{2} d\langle W\rangle_{r}\right)$ and the fact that the empty set is the only subset of $\mathbb{S}$ having zero capacity since $\mathcal{F}(\mathbb{S}) \subseteq C(\mathbb{S})$. Hence, $\int Z_{r} d W_{r}$ is a $\mathbb{P}_{x}$-martingale for all $x \in \mathbb{S}$. Moreover, it follows from the Girsanov theorem that $\left\{Y_{t}\right\}_{t \geq 0}$ is a $\tilde{\mathbb{P}}_{x}$-martingale, and therefore,

$$
u^{n}(T, x)=Y_{0}=\tilde{\mathbb{E}}_{x}\left(Y_{0}\right)=\tilde{\mathbb{E}}_{x}\left(Y_{\sigma^{(T)}}\right)=\tilde{\mathbb{E}}_{x}\left(\Psi\left(\sigma^{(T)}, X_{\sigma^{(T)}}\right)\right),
$$

which, together with the fact that $|\Psi| \leq\|\psi\|_{L^{\infty}}$, implies that $\left\|u^{n}(T)\right\|_{L^{\infty}} \leq\|\psi\|_{L^{\infty}}$. Hence, we conclude that $\left\|u^{n}\right\|_{L^{\infty}\left(0, T ; L^{\infty}\right)} \leq\|\psi\|_{L^{\infty}}$, and that the sequence $\left\{u^{n}\right\}$ is well-defined.

Now, by Theorem 4.16,

$$
\left\|u^{n}\right\|_{L^{2}(0, T ; \mathcal{F})}+\left\|\partial_{t} u^{n}\right\|_{L^{2}\left(0, T ; \mathcal{F}^{-1}\right)} \leq C T, \quad n \in \mathbb{N}
$$

where $C>0$ is a generic constant depending only on $\|\psi\|_{L^{\infty}}$ which may vary on different occasions. Therefore, there exists a subsequence $\left\{u^{n_{k}}\right\}$ and a $u \in$ $L^{2}\left(0, T ; \mathcal{F}\left(\mathbb{S} \backslash \mathrm{V}_{0}\right)\right)$ such that $\partial_{t} u \in L^{2}\left(0, T ; \mathcal{F}^{-1}(\mathbb{S})\right)$, and

$$
\begin{aligned}
\lim _{k \rightarrow \infty} u^{n_{k}} & =u, \quad \text { weakly in } L^{2}\left(0, T ; \mathcal{F}\left(\mathbb{S} \backslash \mathrm{V}_{0}\right)\right), \\
\lim _{k \rightarrow \infty} \partial_{t} u^{n_{k}} & =\partial_{t} u, \quad \text { weakly in } L^{2}\left(0, T ; \mathcal{F}^{-1}(\mathbb{S})\right) .
\end{aligned}
$$

Since $\left\|u^{n}\right\|_{L^{\infty}\left(0, T ; L^{\infty}\right)} \leq\|\psi\|_{L^{\infty}}$, the sequence $\left\{u^{n} \nabla u^{n}\right\}_{n \in \mathbb{N}_{+}}$is bounded in $L^{2}\left(0, T ; L^{2}(\mu)\right)$. By considering a subsequence of $\left\{u^{n_{k}}\right\}$ if necessary, we may assume that $\left\{u^{n_{k}} \nabla u^{n_{k}}\right\}$ is weakly convergent in $L^{2}\left(0, T ; L^{2}(\mu)\right)$. By the uniqueness of weak limits,

$$
\lim _{k \rightarrow \infty} u^{n_{k}} \nabla u^{n_{k}}=u \nabla u, \quad \text { weakly in } L^{2}\left(0, T ; L^{2}(\mu)\right) .
$$

Thus, it follows readily from (5.4)-(5.6) that $u$ is a weak solution to (5.1). Moreover, the estimate $\|u\|_{L^{\infty}\left(0, T ; L^{\infty}\right)} \leq\|\psi\|_{L^{\infty}}$ follows as a corollary of the inequalities $\left\|u^{n}\right\|_{L^{\infty}\left(0, T ; L^{\infty}\right)} \leq\|\psi\|_{L^{\infty}}$.

Testing (5.1) against $u(t)$ and using the Sobolev inequality (3.22) gives that for any $\epsilon \in(0,1)$ and a.e. $t \in[0, T]$,

$$
\frac{d}{d t}\|u(t)\|_{L^{2}(v)}^{2} \leq-\mathcal{E}(u(t))+\|\psi\|_{L^{\infty}}\left[\epsilon \mathcal{E}(u(t))^{1 / 2}+C_{\epsilon}\|u(t)\|_{L^{2}(v)}\right] \mathcal{E}(u(t))^{1 / 2} .
$$

Choosing $\epsilon>0$ sufficiently small gives that

$$
\frac{d}{d t}\|u(t)\|_{L^{2}(v)}^{2} \leq-\frac{1}{2} \mathcal{E}(u(t))+C\|u(t)\|_{L^{2}(v)}^{2}, \text { a.e. } t \in[0, T],
$$

from which the estimate (5.2) follows readily. 
Uniqueness. Suppose that $\bar{u}$ is also a weak solution to (5.1). Then $\|u\|_{L^{\infty}\left(0, T ; L^{\infty}\right)}+$ $\|\bar{u}\|_{L^{\infty}\left(0, T ; L^{\infty}\right)} \leq 2\|\psi\|_{L^{\infty}}$. For any $\epsilon>0$, testing the equation for $u(t)-\bar{u}(t)$ against $u(t)-\bar{u}(t)$ itself gives that

$$
\begin{aligned}
\frac{d}{d t}\|u(t)-\bar{u}(t)\|_{L^{2}(v)}^{2} \leq & -\mathcal{E}(u(t)-\bar{u}(t))+C \| u(t) \\
& -\bar{u}(t) \|_{L^{2}(\mu)}\left[\mathcal{E}(u(t))^{1 / 2}+\mathcal{E}(u(t)-\bar{u}(t))^{1 / 2}\right],
\end{aligned}
$$

where, as before, $C>0$ is a generic constant depending only on $\|\psi\|_{L^{\infty}}$. For any $\epsilon \in(0,1)$, using the Sobolev inequality (3.22), we deduce that

$$
\frac{d}{d t}\|u(t)-\bar{u}(t)\|_{L^{2}(v)}^{2} \leq \frac{C}{\epsilon}\|u(t)-\bar{u}(t)\|_{L^{2}(v)}^{2}+C(1+\epsilon)[\mathcal{E}(u(t))+\mathcal{E}(\bar{u}(t))] .
$$

Therefore,

$$
\|u(t)-\bar{u}(t)\|_{L^{2}(v)}^{2} \leq C(1+\epsilon) \int_{0}^{t} e^{-C(t-s) / \epsilon}[\mathcal{E}(u(s))+\mathcal{E}(\bar{u}(s))] d s .
$$

By the dominated convergence theorem, setting $\epsilon \rightarrow 0$ in the above gives that $\| u(t)-$ $\bar{u}(t) \|_{L^{2}(v)}=0, t \in[0, T]$, which proves the uniqueness.

We now turn to the proof of the joint Hölder continuity. Let $g(t)=u(t) \nabla u(t)$. Then $|g(t)| \leq\|\psi\|_{L^{\infty}}|\nabla u(t)|$. By an argument similar to the proof of (4.21) in Theorem 4.16, we may show that $\|g\|_{L^{\infty}\left(0, T ; L^{2}(\mu)\right)}<\infty$. Since $u$ is the unique solution to

$$
\left\{\begin{array}{c}
\partial_{t} u d v=\mathcal{L} u d v+g(t) d \mu, \quad \text { in }(0, T] \times\left(\mathbb{S} \backslash \mathrm{V}_{0}\right), \\
u=0 \text { on }(0, T] \times \mathrm{V}_{0}, \quad u(0)=\psi,
\end{array}\right.
$$

we may now apply Lemma 4.11 and Proposition 4.15 to obtain the desired joint Hölder continuity.

Acknowledgements The authors would like to thank Professor Ben Hambly for inspiring discussions and providing with many invaluable suggestions on this work. We also want to express our appreciation to Professor Masanori Hino, Dr. Michael Hinz and Professor Alexander Teplyaev for their helpful comments and discussions.

Open Access This article is distributed under the terms of the Creative Commons Attribution 4.0 International License (http://creativecommons.org/licenses/by/4.0/), which permits unrestricted use, distribution, and reproduction in any medium, provided you give appropriate credit to the original author(s) and the source, provide a link to the Creative Commons license, and indicate if changes were made.

\section{References}

1. Barlow, M., Hambly, B.: Transition sensity estimates for Brownian motion on scale irregular Sierpinski gaskets. In: Annales de l'Institut Henri Poincare (B) Probability and Statistics, vol. 33, pp. 531-557. Elsevier (1997)

2. Barlow, M., Kigami, J.: Localized eigenfunctions of the Laplacian on p.c.f self-similar sets. J. Lond. Math. Soc. 56(02), 320-332 (1997) 
3. Barlow, M., Perkins, E.: Brownian motion on the Sierpinski gasket. Probab. Theory Relat. Fields 79(4), 543-623 (1988)

4. Baudoin, F., Kelleher, D.: Differential forms on Dirichlet spaces and Bakry-Émery estimates on metric graphs. Preprint arXiv:1604.02520, (2016)

5. Caffarelli, L., Kohn, R., Nirenberg, L.: First order interpolation inequalities with weights. Compos. Math. 53(3), 259-270 (1984)

6. Cipriani, F., Sauvageot, J.-L.: Derivations as square roots of Dirichlet forms. J. Funct. Anal. 201(1), 78-120 (2003)

7. Evans, L.: Partial Differential Equations, volume 19 of Graduate Studies in Mathematics. American Mathematical Society, 2nd edn (2010)

8. Fitzsimmons, P., Hambly, B., Kumagai, T.: Transition density estimates for Brownian motion on affine nested fractals. Commun. Math. Phys. 165(3), 595-620 (1994)

9. Goldstein, S.: Random walks and diffusions on fractals. In: Percolation Theory and Ergodic Theory of Infinite Particle Systems, pp. 121-129. Springer, New York (1987)

10. Grigor'yan, A., Hu, J., Lau, K.-S.: Heat kernels on metric measure spaces and an application to semilinear elliptic equations. Trans. Am. Math. Soc. 355(5), 2065-2095 (2003)

11. Hambly, B.: Brownian motion on a homogeneous random fractal. Probab. Theory Relat. Fields 94(1), 1-38 (1992)

12. Hambly, B.: Brownian motion on a random recursive Sierpinski gasket. Ann. Probab. 25(3), 1059-1102 (1997)

13. Hino, M.: Martingale dimensions for fractals. Inst. Math. Stat. 36(3), 971-991 (2008)

14. Hino, M.: Energy measures and indices of Dirichlet forms, with applications to derivatives on some fractals. Proc. Lond. Math. Soc. 100(1), 269-302 (2010)

15. Hino, M.: Upper estimate of martingale dimension for self-similar fractals. Probab. Theory Relat. Fields 156(3-4), 739-793 (2013)

16. Hinz, M., Teplyaev, A.: Finite energy coordinates and vector analysis on fractals. Fractal Geom. Stoch. V 70, 209-227 (2015)

17. Hinz, M., Rogers, L.: Magnetic fields on resistance spaces. Preprint arXiv:1501.01100, (2015)

18. Hinz, M., Teplyaev, A.: Dirac and magnetic Schrödinger operators on fractals. J. Funct. Anal. 265(11), 2830-2854 (2013)

19. Hinz, M., Röckner, M., Teplyaev, A.: Vector analysis for Dirichlet forms and quasilinear PDE and SPDE on metric measure spaces. Stoch. Process. Their Appl. 123(12), 4373-4406 (2013)

20. Ionescu, M., Rogers, L., Teplyaev, A.: Derivations, Dirichlet forms and spectral analysis. J. Funct. Anal. 263(8), 2141-2169 (2012)

21. Kigami, J., Lapidus, M.L.: Weyl's problem for the spectral distribution of Laplacians on p.c.f. selfsimilar fractals. Commun. Math. Phys. 158(1), 93-125 (1993)

22. Kigami, J.: A harmonic calculus on the Sierpinski spaces. Jpn. J. Appl. Math. 6(2), 259-290 (1989)

23. Kigami, J.: Harmonic metric and Dirichlet form on the Sierpinski gasket. Pitman Research Notes in Mathematics Series, pp. 201-218, (1993)

24. Kigami, J.: Analysis on Fractals, volume 143 of Cambridge Tracts in Mathematics. Cambridge University Press, Cambridge (2001)

25. Kusuoka, S.: Dirichlet forms on fractals and products of random matrices. Res. Inst. Math. Sci. 25(4), 659-680 (1989)

26. Kusuoka, S.: A diffusion process on a fractal. In: Kiyosi Itô and Nobuyuki Ikeda, editors, Probabilistic Methods in Mathematical Physics: Proceedings of the Taniguchi International Symposium (Katata and Kyoto, 1985), pp. 251-274. Academic Press, (1987)

27. Liu, X., Qian, Z.: Backward problems for stochastic differential equations on the Sierpinski gasket. Stoch. Process. Their Appl. 128(10), 3387-3418 (2018)

28. Mandelbrot, B.: Fractals: Form, Chance and Dimension. W. H. Freeman \& Co., Ltd., San Francisco (1977)

29. Peng, S.: Probabilistic interpretation for systems of quasilinear parabolic partial differential equations. Stoch. Stoch. Rep. 37(1-2), 61-74 (1991)

30. Strichartz, R.: Taylor approximations on Sierpinski gasket type fractals. J. Funct. Anal. 174(1), 76-127 (2000)

31. Strichartz, R.: Function spaces on fractals. J. Funct. Anal. 198(1), 43-83 (2003) 
32. Teplyaev, A.: Spectral analysis on infinite Sierpiński gaskets. J. Funct. Anal. 159(2), 537-567 (1998) 33. Teplyaev, A.: Gradients on fractals. J. Funct. Anal. 174, 128-154 (2000)

Publisher's Note Springer Nature remains neutral with regard to jurisdictional claims in published maps and institutional affiliations. 